\title{
Multi-wavelength Observations of the 2015 Nova in the Local Group Irregular Dwarf Galaxy IC 1613
}

\author{
S. C. Williams, ${ }^{1,2 \star ~ M . ~ J . ~ D a r n l e y, ~}{ }^{2} \dagger$ and M. Henze ${ }^{3} \ddagger$ \\ ${ }^{1}$ Physics Department, Lancaster University, Lancaster, LA1 $4 Y B$, UK \\ ${ }^{2}$ Astrophysics Research Institute, Liverpool John Moores University, IC2 Liverpool Science Park, Liverpool, L3 5RF, UK \\ ${ }^{3}$ Institute of Space Sciences (IEEC-CSIC), Campus UAB, Carrer de Can Magrans, s/n 08193 Barcelona, Spain
}

Accepted XXX. Received YYY; in original form ZZZ

\begin{abstract}
A nova in the Local Group irregular dwarf galaxy IC 1613 was discovered on 2015 September 10 and is the first nova in that galaxy to be spectroscopically confirmed. We conducted a detailed multi-wavelength observing campaign of the eruption with the Liverpool Telescope, the LCO $2 \mathrm{~m}$ telescope at Siding Spring Observatory, and Swift, the results of which we present here. The nova peaked at $M_{V}=-7.93 \pm 0.08$ and was fast-fading, with decline times of $t_{2(V)}=13 \pm 2$ and $t_{3(V)}=26 \pm 2$ days. The overall light curve decline was relatively smooth, as often seen in fast-fading novae. Swift observations spanned 40 days to 332 days post-discovery, but no X-ray source was detected. Optical spectra show the nova to be a member of the hybrid spectroscopic class, simultaneously showing Fe II and N II lines of similar strength during the early decline phase. The spectra cover the eruption from the early optically thick phase, through the early decline and into the nebular phase. The $\mathrm{H} \gamma$ absorption minimum from the optically thick spectrum indicates an expansion velocity of $1200 \pm 200 \mathrm{~km} \mathrm{~s}^{-1}$. The FWHM of the $\mathrm{H} \alpha$ emission line between 10.54 and 57.51 days post-discovery shows no significant evolution and remains at $\sim 1750 \mathrm{~km} \mathrm{~s}^{-1}$, although the morphology of this line does show some evolution. The nova appears close to a faint stellar source in archival imaging, however we find the most likely explanation for this is simply a chance alignment.
\end{abstract}

Key words: novae, cataclysmic variables - stars: individual (Nova IC 1613 2015) ultraviolet: stars

\section{INTRODUCTION}

Classical novae (CNe) are binary systems (Walker 1954; Kraft 1964) with a white dwarf (WD) accreting matter from a non-degenerate companion star (either main-sequence, sub-giant or red giant; see e.g. Darnley et al. 2012). As material builds up on the surface of the WD the pressure and temperature increase until nuclear fusion occurs, leading to a thermonuclear runaway (Starrfield et al. 1972). This causes a rapid increase in luminosity, with the most luminous CN eruptions exceeding $M_{V}=-10$ (Shafter et al. 2009, Williams et al. in prep). By definition, novae with one observed eruption are classified as $\mathrm{CNe}$; those with two or more observed eruptions are classified as recurrent novae (RNe). The shortest recurrence period observed to date is one year, in M31N 2008-12a (see e.g. Darnley et al. 2016). For detailed

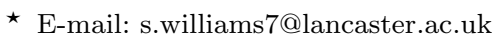

$\dagger$ E-mail: M.J.Darnley@ljmu.ac.uk

† E-mail: henze@ice.cat
}

reviews of the nova phenomenon see Bode \& Evans (2008) and Woudt \& Ribeiro (2014).

Novae have long been considered as potential Type Ia supernova (SN Ia) progenitor candidates (e.g. Whelan \& Iben 1973), with the latest models indicating that WDs in nova systems can indeed gain mass over a long series of eruption cycles and eventually produce a SN Ia (Hillman et al. 2016). While it is widely accepted that SNe Ia are caused by thermonuclear explosions of carbon-oxygen WDs (Hoyle \& Fowler 1960; Nomoto et al. 1984; Hillebrandt \& Niemeyer 2000; Nugent et al. 2011), the mechanisms via which the WD reaches the critical mass to explode is still unclear (see Maoz et al. 2014 for a detailed review of SN Ia progenitor candidates). Although the production of lithium in nova eruptions has been predicted for some time (e.g. Arnould \& Norgaard 1975; Starrfield et al. 1978; Hernanz et al. 1996), observational evidence has recently been found that novae may contribute the majority of the ${ }^{7} \mathrm{Li}$ in the Galaxy (Tajitsu et al. 2015, 2016; Izzo et al. 2015; Molaro et al. 2016).

While it is generally not possible to study individual ex- 
tragalactic novae in as much detail as their Galactic counterparts, there are several advantages to observing extragalactic novae. The large uncertainties in distance that can be associated with Galactic novae are largely negated when studying extragalactic populations and a better representation is given of an entire galaxy's nova population. Additionally, it enables the studies of novae in different environments, for example the stellar populations of the nearby dwarf galaxies, the Large Magellanic Cloud (LMC) and Small Magellanic Cloud (SMC) are very different from the large spirals like M31 and our own Galaxy.

Many Local Group novae are discovered each year, yet to date detailed studies of individual nova eruptions in the low-metallicity environments typically found in dwarf irregular galaxies have been restricted to the nearby Magellanic Clouds (MCs). Dwarf galaxies of course have low nova rates, with the nova rates of the LMC and SMC calculated to be $2.4 \pm 0.8 \mathrm{yr}^{-1}$ and $0.9 \pm 0.4 \mathrm{yr}^{-1}$ respectively (Mróz et al. 2016). This compares to rates of $65_{-15}^{+16} \mathrm{yr}^{-1}$ in M31 (Darnley et al. 2006), $33_{-8}^{+13} \mathrm{yr}^{-1}$ in M81 (Neill \& Shara 2004), and even as high as $363_{-45}^{+33} \mathrm{yr}^{-1}$ in M87 (Shara et al. 2016). To build a full picture of how the properties of novae depend on the properties of their host galaxy, it is important we study nova eruptions in these dwarf irregulars in as much detail as possible.

IC 1613 is an irregular dwarf galaxy in the Local Group at a distance of approximately $730 \mathrm{kpc}$ (Scowcroft et al. 2013; Menzies et al. 2015). Recent evidence suggests it has a metallicity of about one fifth of Solar, similar to that of the SMC (Garcia et al. 2014; Bouret et al. 2015), and its star formation rate has been constant over time (Skillman et al. 2014). IC 1613 differs from the MCs as it is essentially isolated, whereas the MCs are interacting with the Milky Way (see van den Bergh 2000 for an overview).

A total of three nova candidates have previously been discovered in IC 1613. The first was imaged at $B \simeq 17.5$ on three plates taken on a single night by Walter Baade in 1954 November, having not been visible the night before and no further images were taken that season (Sandage 1971). The second candidate was detected on 1996 October 12, although the eruption time of this candidate is poorly constrained, with the last non-detection being two months prior (Mantegazza et al. 2001). For nine days following October 12, the candidate was seen to decline in brightness (Mantegazza et al. 2001). The third and most recent nova candidate was discovered in 1999 (King et al. 1999), but this was actually a Mira variable (Kurtev et al. 2001).

Nova IC 16132015 (PNV J01044358+0203419) was discovered at $01^{\mathrm{h}} 04^{\mathrm{m}} 43^{\mathrm{s}} .58+02^{\circ} 03^{\prime} 41^{\prime \prime} .9$ with an unfiltered magnitude of 17.5 on 2015 September 10.48 UT, with nothing visible down to a limiting magnitude of about 18.0 on September 9 (Halevi et al. 2015), by the Lick Observatory Supernova Search (see Filippenko et al. 2001 for further details). After classification as an extragalactic nova (Williams \& Darnley 2015), we conducted optical, near-IR, near-UV and X-ray observations of the eruption, which we present in this paper.

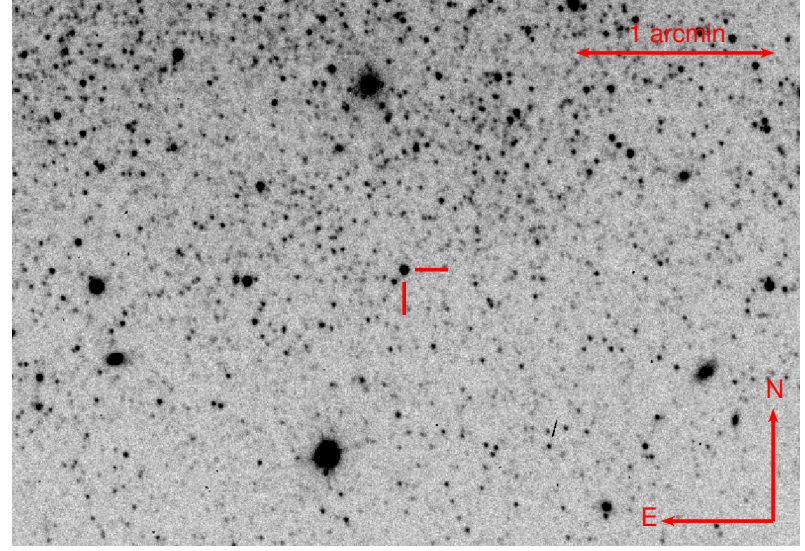

Figure 1. Negative image of Nova IC 16132015 in eruption taken though an $r^{\prime}$-band filter with IO:O on the LT on 2015 Oct 9.00 UT. The position of the nova is indicated by the red lines near the centre of the image.

\section{OBSERVATIONS AND DATA ANALYSIS}

\subsection{Ground-based photometry}

Nova IC 16132015 was initially followed with IO:O ${ }^{1}$, the optical imager on the $2 \mathrm{~m}$ Liverpool Telescope on La Palma, Canary Islands, Spain (LT; Steele et al. 2004), using B, $V$ and $i^{\prime}$ filters, with the first set of observations taken 1.61 days after discovery on 2016 Sep 12.09 UT. Once the nova nature of the object became clear, the filter set was expanded to $u^{\prime}, B, V, r^{\prime}, i^{\prime}$, and $z^{\prime}$. We also began monitoring the eruption in the near-IR using the fixed $H$-band filter on the IO:I imager on the LT (Barnsley et al. 2016). In addition to the LT data, we also obtained some photometric observations through $B, V, r^{\prime}$, and $i^{\prime}$ filters using the Las Cumbres Observatory (LCO) $2 \mathrm{~m}$ telescope at Siding Spring Observatory, New South Wales, Australia (formally the Faulkes Telescope South; FTS, Brown et al. 2013). An IO:O image of the nova in eruption is shown in Figure 1.

The $u^{\prime} B V r^{\prime} i^{\prime} z^{\prime}$ photometry was calculated using aperture photometry in GAIA ${ }^{2}$ and calibrated against field stars from the Sloan Digital Sky Survey Data Release 9 (Ahn et al. 2012). The $B$ and $V$ magnitudes of these calibration stars were calculated using the transformations in Jordi et al. (2006). The $H$-band observations were calibrated against different stars from the 2MASS All Sky Catalog of point sources (Cutri et al. 2003).

\subsection{Spectroscopy}

The optical spectra were taken using the SPectrograph for the Rapid Acquisition of Transients (SPRAT), a lowresolution high-throughput spectrograph on the LT (Piascik et al. 2014). It has a $1^{\prime \prime} .8$ slit width, giving a resolution of $18 \AA$. Our observations were all taken using the blue-

1 http://telescope.livjm.ac.uk/Telinst/Inst/I00

2 GAIA is a derivative of the Skycat catalogue and image display tool, developed as part of the VLT project at ESO. Skycat and GAIA are free software under the terms of the GNU copyright. 
Table 1. Summary of all spectroscopic observations of Nova IC 16132015 with the SPRAT spectrograph on the LT.

\begin{tabular}{lcc}
\hline${\text { Date }[\mathrm{UT}]^{a}}^{a}$ & Days post-discovery & Exposure time [s] \\
\hline 2015 Sep 12.07 & 1.59 & 1800 \\
2015 Sep 17.07 & 6.59 & 1800 \\
2015 Sep 21.02 & 10.54 & 1800 \\
2015 Sep 25.09 & 14.61 & 1800 \\
2015 Oct 07.03 & 26.55 & 3600 \\
2015 Nov 06.99 & 57.51 & 5400 \\
\hline
\end{tabular}

$a$ The date listed here refers to the mid-point of each observation.

optimised mode. The details of the spectra are summarised in Table 1.

Spectrophotometric standards were not observed at similar times as the IC 1613 spectroscopy, but we observed the standard G191-B2B using the same SPRAT instrument set-up on 2015 Dec 17, 2015 Dec 30 and 2016 Jan 10. The flux calibration of each spectrum was performed using standard routines in $\operatorname{IRAF}^{3}$ (Tody 1986). The standard observations were calibrated against data from Oke (1990) obtained via ESO. Due to different observing conditions, and particularly seeing losses and atmospheric conditions (i.e. cloud), the absolute flux calibrations of each spectrum can vary significantly. Examining the standard star observations discussed above, we estimate this causes the typical flux calibration error (at $5000 \AA$ ) to be of order $15-20 \%$. However, the relative calibration across any individual spectrum (i.e. between red and blue, after removing the systematic flux calibration offset) should be relatively good with the uncertainties $<10 \%$.

\section{$2.3 \quad$ Swift observations}

The super-soft X-ray source (SSS) phase in novae is caused by nuclear burning of hydrogen on the surface of the WD. The SSS emission can be detected once the ejecta become optically thin to X-rays and the SSS 'turn-off' is thought to represent the end of nuclear burning (see e.g. Krautter et al. 1996).

We were granted six Swift (Gehrels et al. 2004) target of opportunity (ToO) observations (target ID 34085) to follow the UV and X-ray evolution of the nova. Additionally, we analysed data aimed at IC 1613 itself (target ID 84201), which includes our object in the field of view. All Swift data are summarised in Table 2.

The Swift UV/optical telescope (UVOT, Roming et al. 2005) data were reduced using the HEASoft (v6.16) tool uvotsource. The UVOT magnitudes are based on aperture photometry of carefully selected source and background regions. The photometric calibration assumes the UVOT photometric (Vega) system (Poole et al. 2008) and have not been corrected for extinction. The central wavelengths of the

3 IRAF is distributed by the National Optical Astronomy Observatory, which is operated by the Association of Universities for Research in Astronomy (AURA) under a cooperative agreement with the National Science Foundation. utilised UVOT filters are: $U V W 1: 2600 \AA$; UVM2: $2250 \AA$; UVW2: $1930 \AA$.

All Swift X-ray telescope (XRT; Burrows et al. 2005) data were obtained in the photon counting $(\mathrm{PC})$ mode. For extraction of the count rate upper limits we made use of the on-line interface ${ }^{4}$ of Evans et al. (2009). This tool uses the Bayesian formalism of Kraft et al. (1991) for low numbers of counts. As is recommended for SSSs, only grade zero events were extracted. To convert the counts to Xray fluxes we assume a conservative (maximum) black-body temperature of $50 \mathrm{eV}$ and a Galactic foreground absorption of $N_{\mathrm{H}}=3 \times 10^{20} \mathrm{~cm}^{-2}$. The absorption was derived from the HEASARC $N_{\mathrm{H}}$ tool based on the hydrogen maps of Dickey \& Lockman (1990).

We estimated the X-ray temperature based on the reference frame of the M31 SSS nova sample and the correlations subsequently found by Henze et al. (2014a). In M31, a $t_{2}$ of 13 days (see Section 3.1) would correspond to a SSS phase from about days 60-200, which in turn suggests a blackbody $k T \sim 50 \mathrm{eV}$ (cf. figure 8 of Henze et al. 2014a). Using the pimms software (v4.8c) we estimated an energy conversion factor (count rate divided by unabsorbed flux in the $0.2-1.0 \mathrm{keV}$ band) of $1.2 \times 10^{10} \mathrm{ct} \mathrm{cm}^{2} \mathrm{erg}^{-1}$ for the XRT (PC mode). We derived the corresponding $\mathrm{X}$-ray luminosities in Table 2 by assuming a distance to IC 1613 of $730 \mathrm{kpc}$.

\subsection{Reddening}

IC 1613 is subject to only a small amount of foreground reddening $\left(E_{B-V}=0.021\right.$; Schlafly \& Finkbeiner 2011). However, estimating the reddening internal to IC 1613 at the position of the nova is difficult, as this is highly variable throughout the galaxy (Garcia et al. 2009). In a survey of IC 1613 Cepheid variables, Pietrzyński et al. (2006) found an average total reddening of $E_{B-V}=0.090 \pm 0.019$ to the Cepheids, which we take as the extinction estimate for our absolute magnitude calculations.

\section{RESULTS}

\subsection{Photometric evolution}

A light curve showing all the photometry taken by the LT, LCO $2 \mathrm{~m}$, and Swift is shown in Figure 2. This photometry is also tabulated in Appendix A and presented in the form of spectral energy distributions (SEDs) in Section 3.3. The light curve shows the nova was clearly discovered prior to peak.

The nova follows a relatively uniform decline, although the $r^{\prime}$-band fades significantly slower than $B, V$, and $i^{\prime}$ due to the increasingly strong influence of the $\mathrm{H} \alpha$ emission line on the broadband photometry. Initially the nova also declines more slowly in the $z^{\prime}$-band than other filters, but by 40 days post-discovery, the $z^{\prime}$-band declines more quickly than the other filters. The initial slow $z^{\prime}$ decline is unlikely to indicate a change in the overall nova SED, as the $\left(V-i^{\prime}\right)$ colour evolution remains relatively unaltered during this phase (the early $H$-band observations are also consistent) and therefore the slower $z^{\prime}$ decline is probably line driven. As we have no

\footnotetext{
${ }^{4}$ http://www.swift.ac.uk/user_objects
} 
Table 2. Swift UVOT magnitude and X-ray upper limits.

\begin{tabular}{|c|c|c|c|c|c|c|c|c|c|}
\hline \multirow[t]{2}{*}{ ObsiD } & \multirow{2}{*}{$\begin{array}{r}\operatorname{Exp}^{a} \\
{[\mathrm{ks}]}\end{array}$} & \multirow{2}{*}{$\begin{array}{r}\text { Date }^{b} \\
{[\mathrm{UT}]}\end{array}$} & \multirow{2}{*}{$\begin{array}{r}\mathrm{MJD}^{b} \\
{[\mathrm{~d}]}\end{array}$} & \multirow{2}{*}{$\begin{array}{r}\Delta t^{c} \\
{[\mathrm{~d}]}\end{array}$} & \multicolumn{3}{|c|}{$\mathrm{UV}^{d}[\mathrm{mag}]$} & \multirow{2}{*}{$\begin{array}{r}\text { Rate } \\
{\left[10^{-3} \mathrm{ct} \mathrm{s}^{-1}\right]}\end{array}$} & \multirow{2}{*}{$\begin{array}{c}\mathrm{L}_{0.2-1}^{e}{ }_{0}^{0} \\
{\left[10^{37} \mathrm{erg} \mathrm{s}^{-1}\right]}\end{array}$} \\
\hline & & & & & $U V W 1$ & $U V M 2$ & $U V W 2$ & & \\
\hline 00034085001 & 4.6 & $2015-10-20.54$ & 57315.55 & 40.07 & $18.6 \pm 0.1$ & & & $<4.2$ & $<2.3$ \\
\hline 00034085002 & 4.2 & 2015-11-08.29 & 57334.29 & 58.81 & $19.6 \pm 0.1$ & & & $<2.6$ & $<1.5$ \\
\hline 00034085003 & 4.6 & $2015-11-29.22$ & 57355.23 & 79.75 & $20.2 \pm 0.2$ & & & $<5.5$ & $<3.1$ \\
\hline 00034085004 & 4.1 & $2016-01-08.04$ & 57395.05 & 119.57 & $20.9 \pm 0.3$ & $\ldots$ & $\ldots$ & $<2.1$ & $<1.2$ \\
\hline 00034085005 & 4.0 & 2016-02-09.09 & 57427.09 & 151.61 & $>20.9$ & $\ldots$ & & $<3.2$ & $<1.8$ \\
\hline 00084201006 & 1.3 & $2016-02-17.81$ & 57435.81 & 160.33 & $>19.5$ & $>19.8$ & $>20.0$ & $<8.7$ & $<4.8$ \\
\hline 00084201007 & 6.5 & 2016-05-26.43 & 57534.44 & 258.96 & $>20.1$ & $>20.8$ & $>21.1$ & $<1.8$ & $<1.0$ \\
\hline 00084201008 & 0.9 & 2016-05-28.90 & 57536.90 & 261.42 & $>19.3$ & $>19.6$ & $>19.8$ & $<9.5$ & $<5.3$ \\
\hline 00034085006 & 2.4 & 2016-08-07.07 & 57607.07 & 331.59 & $>21.0$ & $\ldots$ & $\ldots$ & $<4.9$ & $<2.7$ \\
\hline
\end{tabular}

${ }^{a}$ Dead-time corrected XRT exposure time.

$b$ Start date of the observation.

$c$ Time in days after the eruption on 2015-09-10.48 UT (MJD 57275.48).

${ }^{d}$ Vega magnitudes for the $U V W 1, U V M 2$, and $U V W 2$ filters with central wavelength: $2600 \AA, 2250 \AA$, and $1930 \AA$, respectively.

$e$ X-ray luminosity upper limits (unabsorbed, blackbody fit, $0.2-1.0 \mathrm{keV}$ ) were estimated according to Sect. 2.3.

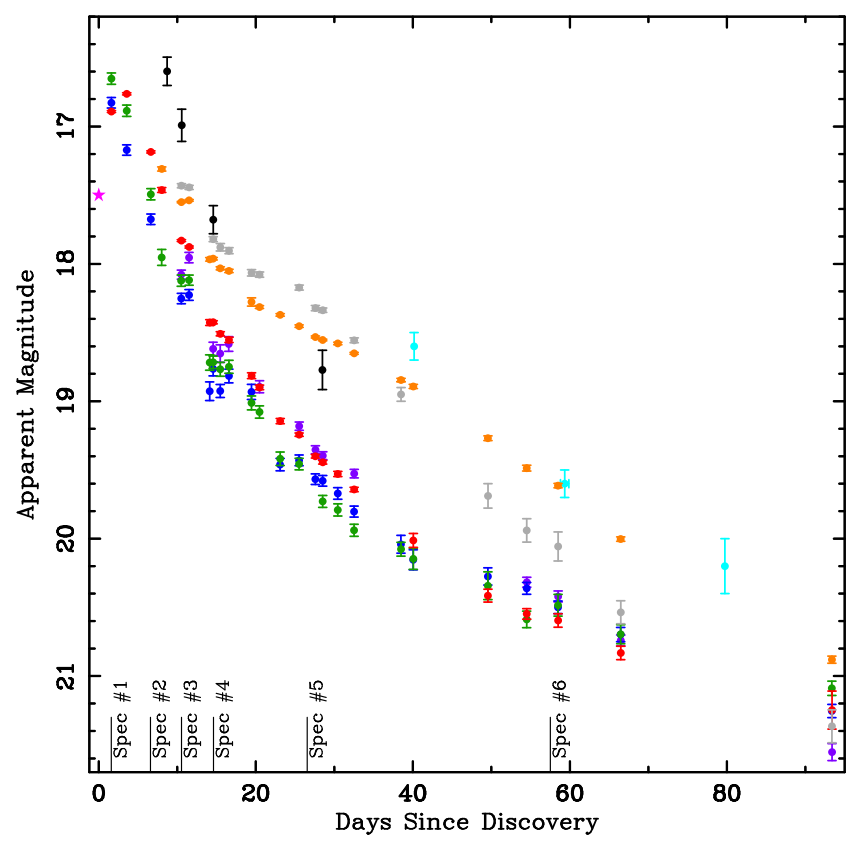

Figure 2. Light curve of Nova IC 1613 2015. The colours represent different filters: $U V W 1$, cyan; $u^{\prime}$, purple; $B$, blue; $V$, green; $r^{\prime}$, orange; $i^{\prime}$, red; $z^{\prime}$, grey; $H$, black. The magenta star shows the unfiltered discovery magnitude. The points on the light curve that correspond to the dates the spectra were taken are also indicated.

spectra extending beyond $8000 \AA$, the species that may be responsible for this is not certain, but we suggest it is most likely due to very strong O I $8446 \AA$ emission (caused by Ly $\beta$ fluorescence; see discussion in Section 3.2).

Adopting a distance modulus $24.31 \pm 0.04$ (weighted average from Scowcroft et al. 2013; Menzies et al. 2015) and correcting for reddening using $E_{B-V}=0.090 \pm 0.019$ (Pietrzyński et al. 2006) and the extinction law from Cardelli et al. $\left(1989, R_{V}=3.1\right)$ gives an absolute magnitude for the eruption peak of $M_{V}=-7.93 \pm 0.08$, which is typical for a nova. The absolute magnitude at 15 days after peak is $M_{V}=-5.84_{-0.10}^{+0.20}$, we note that the (conserva- tive) constraints on the time of peak (assuming 2016 Sep $12.09_{-1.61}^{+1.95}$ UT) dominate the upper error bar. This is similar to that expected from the relationships of the absolute magnitudes of novae 15 (or 17) days after peak brightness in M49 $\left(M_{V, 15}=-6.36 \pm 0.19 ;\right.$ Ferrarese et al. 2003) and M87 $\left(M_{F 606 W, 17}=-6.06 \pm 0.23\right.$; Shara et al. 2017 , although note the 'wide $V$ ' F606W filter contains $\mathrm{H} \alpha$ ). We measure the dereddened day- 15 colour, $(B-V)_{t=15}=-0.03_{-0.14}^{+0.11}$. In the SDSS filters we find $M_{r^{\prime}, 15}=-6.50_{-0.09}^{+0.17},\left(u^{\prime}-r^{\prime}\right)_{t=15}=0.34_{-0.09}^{+0.14}$, $\left(r^{\prime}-i^{\prime}\right)_{t=15}=-0.56 \pm 0.05$ and $\left(i^{\prime}-z^{\prime}\right)_{t=15}=0.60_{-0.05}^{+0.08}$. The $H$-band coverage is much poorer than the other filters, but using extrapolation we estimate $M_{H, 15}=-6.53_{-0.20}^{+0.22}$.

Taking the brightest data point as the peak magnitude of the nova, and using linear extrapolation between the data points, we estimate the $t_{2}$ of this nova to be $15 \pm 3,13 \pm 2$ and $15 \pm 3$ days in $B, V$ and $i^{\prime}$ filters, respectively. We estimate the $t_{3}$ values to be $t_{3(B)}=32 \pm 3, t_{3(V)}=26 \pm 2$ and $t_{3\left(i^{\prime}\right)}=$ $32 \pm 3$ days (the uncertainties here are largely due to the cadence around peak).

\subsection{Spectroscopic Evolution}

Novae have been observed spectroscopically for 150 years, since the first eruption of RN T Coronae Borealis in 1866 (Huggins 1866). Nova spectra tend to fit into one of two groups, named after the dominant non-Balmer species in the spectra, Fe II and He/N classes (Williams 1992). These two types of spectra are suggested to form in different components of gas, with the spectral type observed for a given nova reflecting the dominant spectral component at that time (Williams 2012). The Fe II spectra have been suggested to originate in the circumbinary gas originating from the companion star, whereas the He/ $\mathrm{N}$ type is suggested to be produced by the ejecta themselves (Williams 2012). Although there are some exceptions, Fe II novae tend to produce narrower emission lines than $\mathrm{He} / \mathrm{N}$ novae (see e.g. Williams 1992; Shafter et al. 2011). Line identification can be difficult in novae due to the broad lines and often differing line profiles. This is further complicated when using low-resolution spectra, which are often required to study faint extragalactic novae. However, the multiple epochs of spectra we have 
obtained allow us to identify some lines that may not have been possible with a single observation, and more importantly, better interpret the overall evolution. Line identification was also significantly aided by the extensive nova line list from Williams (2012) and multiplet tables from Moore (1945). The spectra are shown in Figures 3, 4, and 5. All spectra are shown in the frame of the observer, but when discussing the identification of spectroscopic features, rest-frame wavelengths are used. The average radial velocity of IC 1613 is $-231.6 \mathrm{~km} \mathrm{~s}^{-1}$, with a velocity dispersion of $10.8 \mathrm{~km} \mathrm{~s}^{-1}$ (Kirby et al. 2014).

\subsubsection{Optically thick 'fireball' stage}

Our first spectrum was taken on 2015 Sep 12.07, 1.59 days after discovery, and around peak brightness. The main features of this spectrum are the Balmer lines with clear P Cygni absorption profiles. $\mathrm{H} \alpha$ is seen mainly in emission with a small, blue-shifted absorption component. $\mathrm{H} \beta$ is seen with significant emission and absorption components, with $\mathrm{H} \gamma$ and $\mathrm{H} \delta$ mainly detected in absorption. This optically thick spectrum is shown in Figure 3. Fitting a Gaussian to the $\mathrm{H} \gamma$ absorption profile and taking into account the radial velocity of IC 1613 itself, the absorption minimum implies a velocity of $1200 \pm 200 \mathrm{~km} \mathrm{~s}^{-1}$. Fe II $5169 \AA$ is seen mainly in absorption, with features corresponding to the Fe II (42) triplet at 4924 and $5018 \AA$ also tentatively detected. A few other weak absorption lines are also seen, e.g. one at $4465 \AA$ from $\mathrm{Mg}$ II/Fe II.

\subsubsection{Early decline}

The second spectrum, taken 6.59 days after discovery, shows a dramatic change from the $t=1.59$ day spectrum and clearly shows the nova nature of the transient, indeed this is the spectrum we used to announce that the transient was a nova eruption in Williams \& Darnley (2015). This, along with the third, fourth and fifth spectra, taken at $t=10.54$, 14.61 and 26.55 days, respectively, are shown in Figure 4. In the second spectrum, the nova now shows strong Balmer emission, although weak P Cygni absorption components are still present. The Fe II (42) triplet, only just detected in the first spectrum is now clearly seen in emission. In the early decline spectra of novae, triplet 42 is typically the strongest of the Fe II lines, although several other multiplets, located between $\mathrm{H} \alpha$ and $\mathrm{H} \beta$, are usually easily identifiable in regular Fe II novae (e.g. 48, 49, 55, and 74). The Fe II (48) multiplet is the only one of these that appears to be weakly detected in Nova IC 1613 2015, with multiplets 49, 55, and 74 not detected.

In the $t=6.59$ day spectrum, the $\mathrm{N}$ I (3) triplet is seen strongly in emission. It also shows a relatively broad absorption component, as would be expected given the wavelengths of the lines that make up the triplet (7424, 7442, and $7468 \AA$ ), the velocities associated with the nova and the resolution of the spectrograph. The $\mathrm{N}$ II (3) multiplet around $5682 \AA$ is seen with a very strong absorption component, with the N II (28) multiplet at $5938 \AA$ also identified. The profile at the position of the Fe II $5018 \AA$ component of triplet 42 , clearly has a different morphology than the $5169 \AA$ line, the former having a strong absorption component. We interpret this as indicating the presence of N II $5001 \AA$. This is consistent with the morphology of the N II (3) multiplet, which also shows a very strong absorption component. It also explains the evolution of the $5018 \AA$ line profile between spectra two and five (see below). The Bowen blend (N III/C III/O II; this complex is discussed by Harvey et al. in prep), which is sometimes referred to as '4640 emission' (it is at $\sim 4640 \AA$ ) is detected as a broad emission line with an accompanying broad absorption profile. This complex is visible at the time of the emergence of the nebular lines in most novae, however is typically only visible in the early spectra if the nova is a member of the $\mathrm{He} / \mathrm{N}$ spectroscopic class.

The strongest non-Balmer line visible in the $t=6.59$ day spectrum is O I $7774 \AA$, produced by O I (1) triplet. There is a relatively strong emission line peaking at $6162 \AA$ (again with an absorption profile). This is clearly not the Fe II (74) multiplet emission line at $6148 \AA$ as the other lines are not present (notably the $6248 \AA$ line, for example). We identify this as most likely being the O I (10) triplet at $6157 \AA$. Alternatively it could be N II (which has lines at a similar wavelength), although that is perhaps less likely given it fades to be undetected by the fifth spectrum, which is very different from the other three N II lines (5001, 5682 and $5938 \AA$ ), which remain detected even in the final nebular spectrum, as discussed below. In the second spectrum, there is an emission line peaking at $6722 \AA$. An emission line at this wavelength has been noted since the early days of nova spectroscopy (e.g. DN Geminorum; Wright 1940) and has been suggested as O I $6726 \AA$ (e.g. Baschek 1964, Mason et al. 2005b, Shore et al. 2013, Munari et al. 2014). An alternative explanation would be the N I $6723 \AA$ line.

The Fe II $5018 \AA / \mathrm{N}$ II $5001 \AA$ emission line appears to extend further redward than expected from other lines, which indicates the presence of another emission line. There is a He I $5048 \AA$ line which is a possible explanation, but there are no He I lines at 6678 or $7065 \AA$, so a more likely explanation is N II. In the second and third spectra there is a weak feature at $\sim 7110 \AA$, which we identify as C I. We also identify C II emission at 4267 and $7234 \AA$, which is visible from the day 6.59 to 26.55 spectra.

The spectroscopic evolution between day 6.59 and 26.55 shows the P Cygni profiles, that initially accompany many of the emission lines, weaken over time, as is usually seen in nova eruptions. The Fe II lines weaken along with the N I and O I lines (although O I $7774 \AA$ is still easily visible in the day 26.55 spectrum). The N II and $\mathrm{N}$ III lines retain their strength through this evolution and by day 26.55, apart from the Balmer and O I $7774 \AA$ lines, the spectrum is essentially dominated by ionised nitrogen lines (from the point of view of visible features, not the overall flux of the spectrum). The contrasting evolution of the Fe II/N II lines can be seen in the morphology of the blended line due to N II $5001 \AA$ and Fe II $5018 \AA$, where the blue side of the blend become increasingly dominant as the nova evolves.

\subsubsection{Nebular phase}

Very few novae beyond the MCs have been observed spectroscopically in the nebular phase. As the evolution progresses, nebular lines also begin to emerge with [OI] (6300 and $6364 \AA$ ) clearly detected by day 14.61 and possibly present 


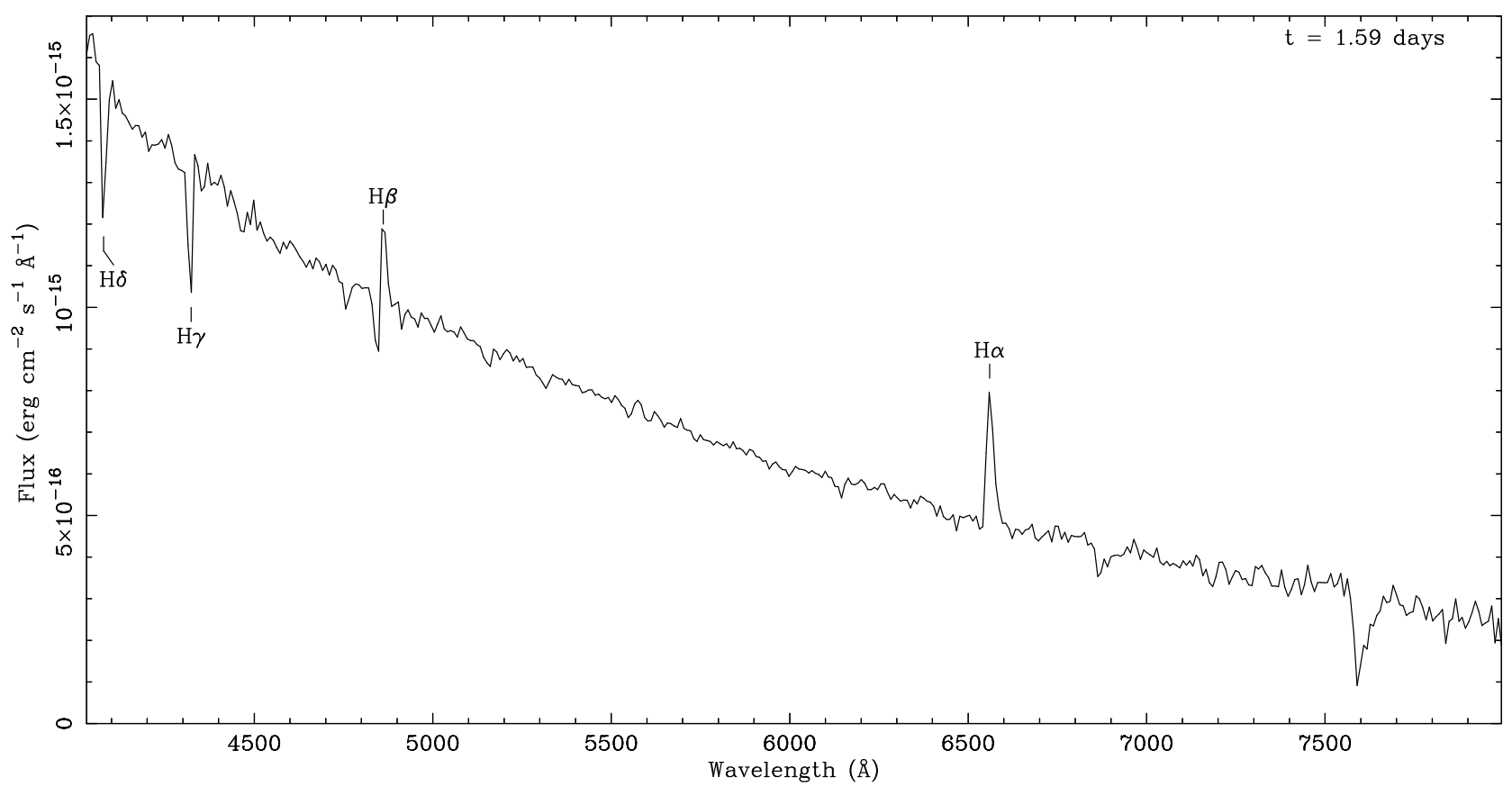

Figure 3. The first spectrum of Nova IC 16132015 taken 2015 Sep 12.07 UT, 1.59 days after discovery, and around peak optical brightness.

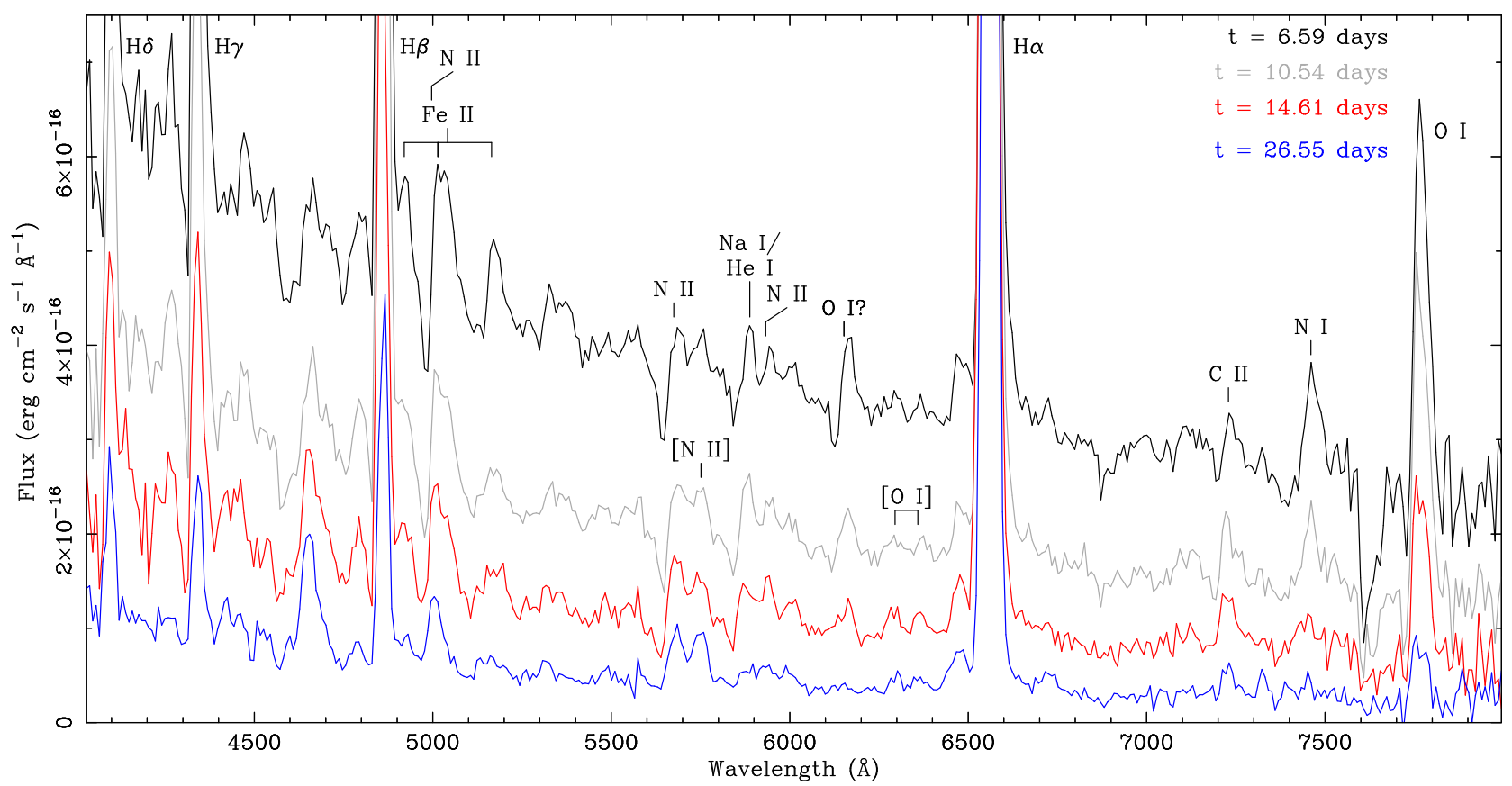

Figure 4. The early decline spectra of Nova IC 1613 2015. These were obtained 2015 Sep 17.07 UT ( $t=6.59$ days; black line), Sep 21.02 $(t=10.54$ days; grey line), Sep $25.09(t=14.61$ days; red line $)$ and Oct $7.03(t=26.55$ days; blue line $)$.

even earlier. The [O II] $7320 / 7330 \AA$ doublet is also likely present in the 26.55 day spectrum. Our sixth and final spectrum was taken 57.41 days after discovery and about 4 magnitudes below peak. This shows further evolution into the nebular phase with [O III] (4959 and $5007 \AA$ ) now clearly visible. The $5007 / 4959 \AA$ emission line ratio is higher than expected (should be $\sim 3$; the lines are highly forbidden and the ratio is essentially fixed), indicating the line is still blended with N II $5001 \AA$. He II (4686 $\AA$ ) can now be seen emerging from the Bowen complex, with the peak of the complex itself consistent with it being dominated by N III. We also find He I emission (5876 and $7065 \AA$ ). This spectrum is shown in Figure 5. The emission line fluxes of prominent lines are shown in Table 3.

The assignment of [N II] $(5755 \AA)$ is correct in the later spectra (e.g. nebular [ $\mathrm{N}$ II] would be expected when [O I] is clearly detected in the $t=14.61$ day spectrum), however there appears to be a line there even in the $t=6.59$ day 


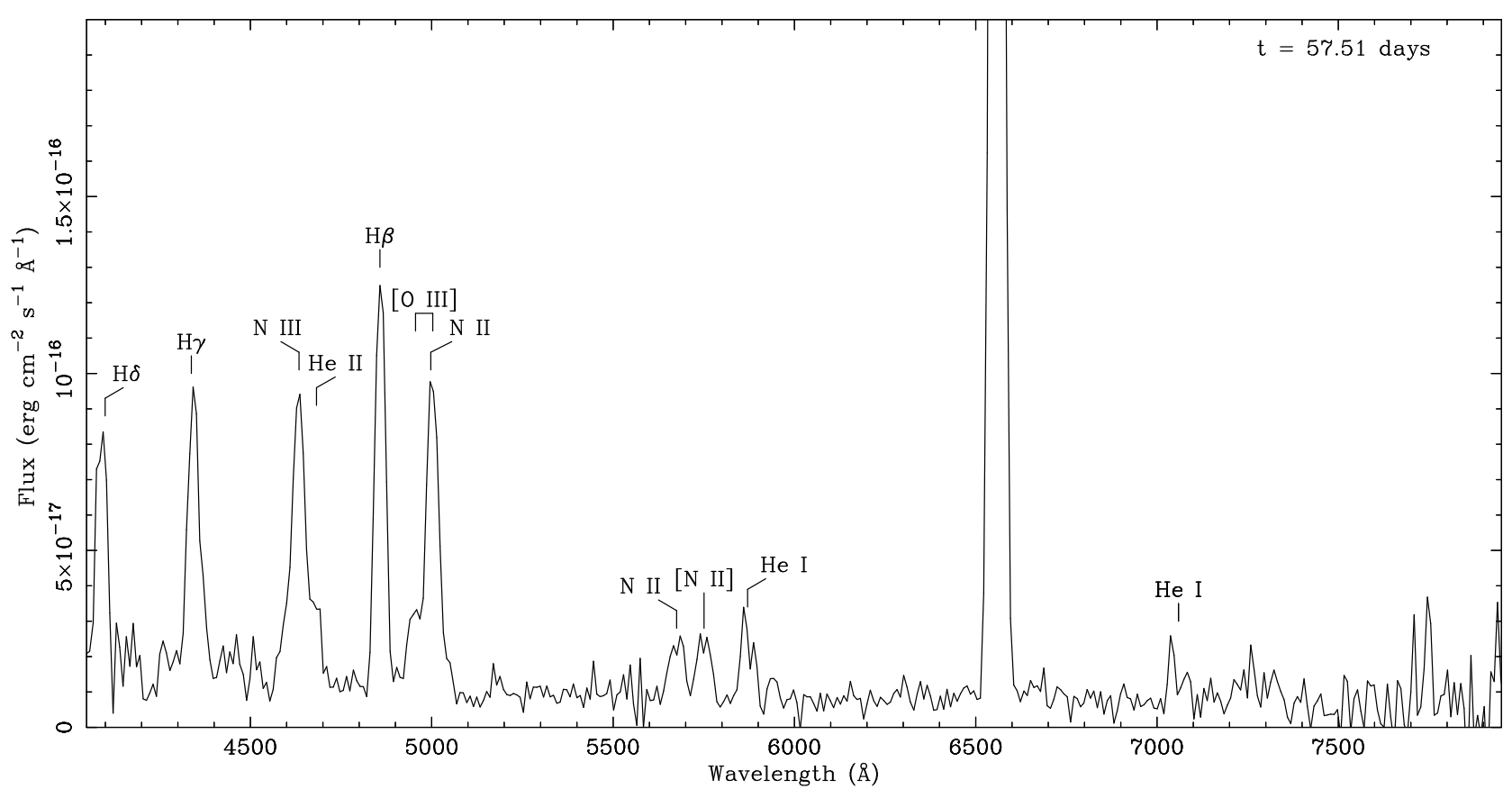

Figure 5. The final spectrum of Nova IC 16132015 taken 2015 Nov 6.99 UT, 57.51 days after discovery. This shows the nova in the nebular phase with strong [O III] emission.

spectrum. This has been noted by other authors (e.g. Iijima \& Esenoglu 2003; Surina et al. 2014). There is also a nonforbidden N II doublet (9) at a very similar wavelength. This is caused by the same excited state as the $\mathrm{N}$ II (3) multiplet, but here the electrons transition to $2 s^{2} 2 p 3 s^{1} \mathrm{P}^{\circ}$, rather than $2 s^{2} 2 p 3 s^{3} \mathrm{P}^{\circ}$. It is possible that this at least partially contributes to this emission line.

\subsubsection{Balmer evolution}

By fitting of a Gaussian profile to the emission component of the $\mathrm{H} \alpha$ line in the second $(t=6.59$ days $)$ spectrum, we measure the FWHM to be $1580 \pm 70 \mathrm{~km} \mathrm{~s}^{-1}$ after correcting for the spectral resolution. The line then apparently broadens to a FWHM of $1750 \pm 50 \mathrm{~km} \mathrm{~s}^{-1}$ in the third $(t=10.54$ day $)$ spectrum and thereafter remains consistent. We measure it at $1760 \pm 90,1750 \pm 120$ and $1720 \pm 190 \mathrm{~km} \mathrm{~s}^{-1}$ in the $t=14.61$, $t=26.55$ and $t=57.51$ day spectra, respectively. The most likely explanation for the early change in the FWHM is that in the $t=6.59$ day spectrum the $\mathrm{H} \alpha$ emission line is significantly influenced by a P Cygni absorption component, which has the effect of narrowing the apparent emission line.

The peaks of the Balmer emission are not shown in Figures 4 and 5 to allow the reader to view the fainter lines in greater detail. The evolution of the $\mathrm{H} \alpha$ line is show in Figure 6. The left panel of Figure 6 shows the overall profile is relatively symmetrical, with a Gaussian profile generally fitting the central profile well. The only stage when a Gaussian does not appear a good fit is the $t=26.65$ day spectrum, when the profile seems asymmetric, being stronger at the red side of the profile. Close inspection of the $\mathrm{H} \alpha$ profile in the right panel of Figure 6 shows there is emission peaking at around $-4000 \mathrm{~km} \mathrm{~s}^{-1}(\sim 6480 \AA)$. Comparing it to the red side of the $\mathrm{H} \alpha$ line shows it is too blue to be explained as part of a simple Gaussian with a P Cygni profile superimposed on the emission component, and could be due to emission from N I or N II. An alternative explanation could be a separate higher velocity component to the $\mathrm{H} \alpha$ line as there may be excess flux on the red side of the profile as well, although this $\sim 6480 \AA$ emission appears to persist longer, visible in all but the final nebular spectrum.

In Figure 7 we show the evolution of the $\mathrm{H} \alpha / \mathrm{H} \beta$ ratio between $t=6.59$ and $t=57.51$ days, corrected for Galactic reddening $\left(E_{B-V}=0.021\right)$. The figure shows the ratio initially increases, peaking at $7.5 \pm 1.0$ on $t=26.55$ days, before declining in the final nebular phase spectrum. During this period the $\mathrm{H} \gamma / \mathrm{H} \beta$ ratio does not change dramatically. The evolution in the $\mathrm{H} \alpha / \mathrm{H} \beta$ ratio can clearly not be caused by dust as such a dramatic change would be seen as a dip in the optical light curve. This $\mathrm{H} \alpha / \mathrm{H} \beta$ ratio evolution is common in novae and has been discussed by a number of authors (e.g. Kogure 1961; Meinel 1963; Ferland 1978; Ferland et al. 1979; Anupama et al. 1992; Iijima \& Esenoglu 2003). The changing Balmer decrement is caused by self-absorption. If Ly $\alpha$ and $\mathrm{H} \alpha$ have high optical depth, high $\mathrm{H} \alpha / \mathrm{H} \beta$ ratios such as those observed here can be produced (Netzer 1975). The calculations made by Netzer (1975) also indicate the $\mathrm{H} \gamma / \mathrm{H} \beta$ ratio does not necessarily change dramatically during this $\mathrm{H} \alpha / \mathrm{H} \beta$ evolution, although this is dependent on the Ly $\alpha$ optical depth. This Balmer line ratio behaviour appears to well replicate that observed in Nova IC 1613 2015. In the case of novae in eruption, Case B recombination is not valid (as discussed above), therefore the Balmer decrement cannot, and should not, be used to estimate reddening.

As suggested by Bowen (1947), the close proximity of O I $1025.76 \AA$ to $\operatorname{Ly} \beta(1025.72 \AA)$ can lead to excitation of the $\mathrm{O}$ I ground state. This then produces strong emission at the $1.1287 \mu \mathrm{m}$ and $8446 \AA$ wavelengths as the electrons fall back to the O I ground state (see also Kastner \& Bhatia 1995). This effect and its relationship to the Balmer 
Table 3. The evolution of emission line fluxes.

\begin{tabular}{|c|c|c|c|c|c|c|}
\hline \multirow{2}{*}{$\begin{array}{l}\text { Line identification } \\
\text { (rest wavelength) }\end{array}$} & \multicolumn{6}{|c|}{ Emission line flux $\left[\times 10^{-15} \mathrm{erg} \mathrm{cm}^{-2} \mathrm{~s}^{-1}\right]$} \\
\hline & $t=1.59$ days & $t=6.59$ days & $t=10.54$ days & $t=14.61$ days & $t=26.55$ days & $t=57.51$ days \\
\hline $\mathrm{H} \delta(4102 \AA)$ & $\ldots$ & $18.7 \pm 3.3$ & $9.5 \pm 1.2$ & $7.0 \pm 1.9$ & $4.3 \pm 1.1$ & $3.9 \pm 0.6$ \\
\hline $\mathrm{H} \gamma(4341 \AA)$ & $\ldots$ & $28.5 \pm 5.2$ & $16.6 \pm 3.0$ & $9.0 \pm 1.6$ & $4.9 \pm 0.7$ & $4.4 \pm 0.3$ \\
\hline $\mathrm{H} \beta(4861 \AA)$ & $3.2 \pm 1.6$ & $68.0 \pm 4.6$ & $43.9 \pm 4.0$ & $24.6 \pm 2.9$ & $12.1 \pm 1.2$ & $5.4 \pm 0.3$ \\
\hline Fe II $(5169 \AA)$ & $\ldots$ & $3.8 \pm 0.8$ & $2.7 \pm 0.4$ & $2.7 \pm 0.6$ & $\ldots$ & $\ldots$ \\
\hline N II $(5682 \AA)$ & $\ldots$ & $1.5 \pm 0.5$ & $2.8 \pm 0.7$ & $3.2 \pm 0.5$ & $3.1 \pm 0.3$ & $1.0 \pm 0.2$ \\
\hline [N II] $(5755 \AA$ A $)$ & $\ldots$ & $1.2 \pm 0.2$ & $2.6 \pm 0.3$ & $2.5 \pm 0.4$ & $3.5 \pm 0.3$ & $1.0 \pm 0.2$ \\
\hline N II $(5939 \AA)$ & $\ldots$ & $1.1 \pm 0.2$ & $2.4 \pm 0.6$ & $2.2 \pm 0.3$ & $\ldots$ & $\ldots$ \\
\hline O I $(6157 \AA)$ & $\ldots$ & $2.6 \pm 0.3$ & $1.8 \pm 0.3$ & $1.1 \pm 0.3$ & $\ldots$ & $\ldots$ \\
\hline [O I] $(6300 \AA)$ & $\ldots$ & $\ldots$ & $\ldots$ & $1.0 \pm 0.3$ & $0.6 \pm 0.2$ & $\ldots$ \\
\hline [O I] $(6364 \AA)$ & $\ldots$ & $\ldots$ & 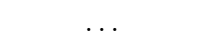 & $0.9 \pm 0.2$ & 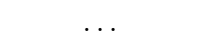 & $\ldots$ \\
\hline $\mathrm{H} \alpha(6563 \AA$ A $)$ & $7.3 \pm 1.1$ & $146.4 \pm 5.1$ & $144.0 \pm 5.2$ & $122.0 \pm 3.7$ & $90.5 \pm 7.4$ & $29.1 \pm 2.1$ \\
\hline C II $(7235 \AA)$ & $\ldots$ & $1.4 \pm 0.7$ & $2.1 \pm 0.7$ & $2.7 \pm 0.4$ & $1.2 \pm 0.3$ & $\ldots$ \\
\hline N I $(7452 \AA)$ & $\ldots$ & $5.4 \pm 0.7$ & $3.2 \pm 0.4$ & $1.6 \pm 0.5$ & $\ldots$ & $\ldots$ \\
\hline O I $(7774 \AA)$ & $\ldots$ & $18.1 \pm 2.2$ & $16.6 \pm 2.7$ & $8.5 \pm 1.7$ & $2.8 \pm 1.2$ & $\ldots$ \\
\hline
\end{tabular}

The emission line fluxes are dependent on the assumed continuum level and if a P Cygni absorption component is present, only the emission component of the feature is measured. The fluxes are dereddened for foreground Galactic extinction, assuming $\boldsymbol{E}_{\boldsymbol{B}-\boldsymbol{V}}=0.021$ and $R_{V}=3.1$. The errors do not take into account uncertainties in the flux calibration discussed in Section 2.2.
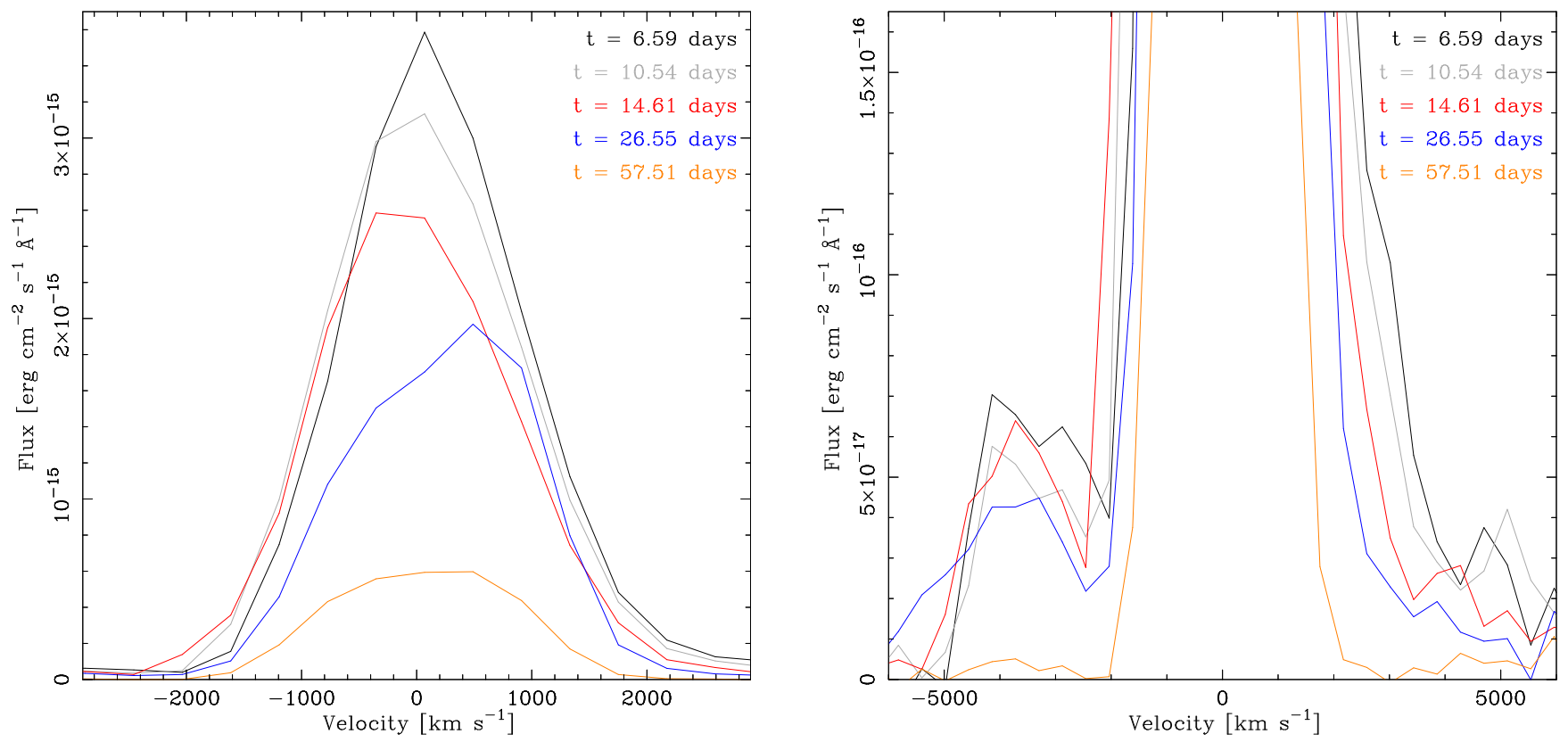

Figure 6. Evolution of the $\mathrm{H} \alpha$ line from $t=6.59$ to $t=57.51$ days. The left panel shows the evolution of the shape and absolute flux of the line. The right panel shows the evolution of the fainter emission either side of the main $\mathrm{H} \alpha$ profile (see discussion in Section 3.2.4). The velocities have been corrected for the radial velocity of IC 1613 itself.

decrement has also been discussed in the context of Seyfert galaxies (Shields 1974). Such Ly $\beta$ florescence can only occur under conditions of optically thick hydrogen. The $\mathrm{H} \alpha / \mathrm{H} \beta$ ratio and the O I $8446 \AA$ intensity are closely linked (Ferland \& Netzer 1979; Ferland et al. 1979), with the $\mathrm{H} \alpha / \mathrm{H} \beta$ and $(\mathrm{O}$ I $8446 \AA) / \mathrm{H} \beta$ line ratios often peaking at a similar point of the eruption (see e.g. Ferland et al. 1986). We note that between the 26.55 and 57.51 day spectra the $\mathrm{H} \alpha / \mathrm{H} \beta$ ratio drops. Between the 26.55 and 57.51 days spectra, it can also be seen from Figure 2 that the $z^{\prime}$-band fades more rapidly than any other waveband, indicating Ly $\beta$ florescence (or specifically the $8446 \AA$ line; as indicated by the drop in the $\mathrm{H} \alpha / \mathrm{H} \beta$ ratio) may be the cause of the initially slower $z^{\prime}$-band decline mentioned in Section 3.1.

\subsection{Spectral energy distributions}

SEDs can be derived from multiband photometry or spectra, both of which have drawbacks. Spectra are more timeexpensive and are more prone to (variable and colourdependent) calibration issues. However, they allow prominent spectral features not associated with the underlying 


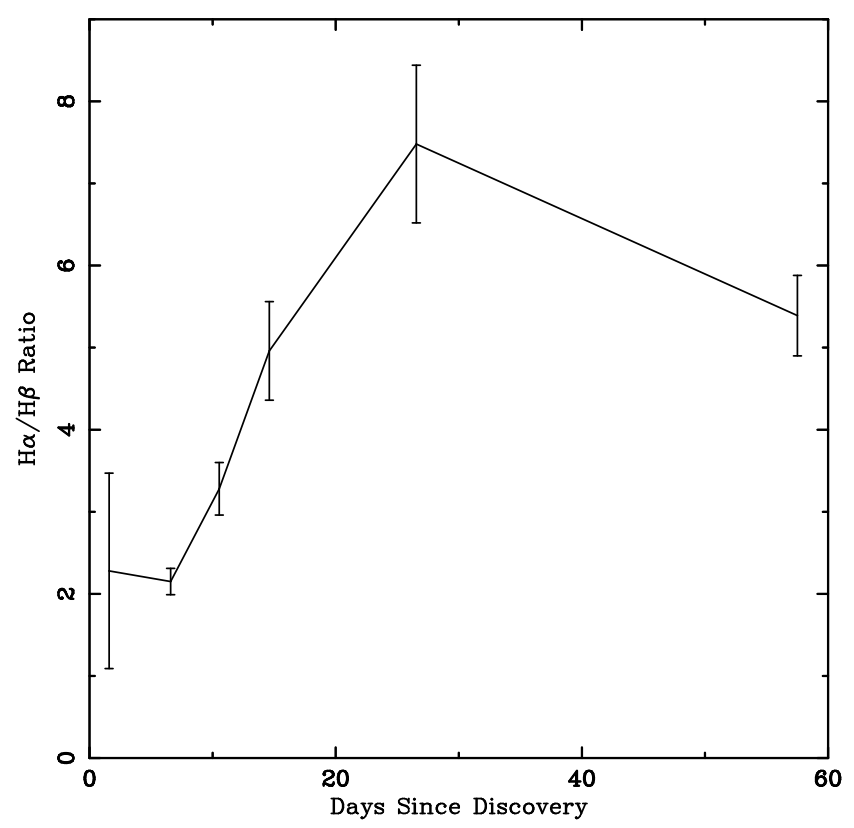

Figure 7. The evolution of the $\mathrm{H} \alpha / \mathrm{H} \beta$ intensity ratio between the $t=6.59$ and $t=57.51$ day spectra. The ratios are corrected for foreground Galactic reddening $\left(E_{B-V}=0.021\right)$.

SED to be removed before fitting, which broadband photometric observations do not. This is particularly important in novae, where during an eruption, the broadband photometry becomes increasingly influenced by line emission and can even be dominated by it at late times (e.g. [O III] and $\mathrm{H} \alpha$ ).

Fitting a power-law to the first spectrum (excluding prominent emission and absorption features) indicates at 1.59 days post-discovery, $f_{\lambda} \propto \lambda^{-2.42 \pm 0.08}$ at optical wavelengths. This is near that expected from optically thick freefree emission $\left(f_{\lambda} \propto \lambda^{-8 / 3}\right.$; Wright \& Barlow 1975). The relatively short wavelength coverage however is also consistent with a black-body. Fitting a black-body function to the first spectrum yields a photospheric temperature of $11600 \pm 500 \mathrm{~K}$. This is close to the expected effective temperature of a nova at peak ( 8000 K; see e.g. Prialnik 1986; Beck et al. 1995; Evans et al. 2005). Five days later the wavelength dependence of the optical continuum had changed dramatically to $f_{\lambda} \propto \lambda^{-1.48 \pm 0.08}$, even shallower than that expected from optically thin free-free $\left(f_{\lambda} \propto \lambda^{-1.9}\right)$. The measured wavelength dependence of the SED increases for the third (10.54 day) spectrum, with $f_{\lambda} \propto \lambda^{-1.58 \pm 0.09}$, although note these are consistent within the errors. The other three spectra give $\propto \lambda^{-1.68 \pm 0.11}, \underset{\sim}{\sim} \lambda^{-2.11 \pm 0.12}$ and $\propto \lambda^{-1.21 \pm 0.18}$ on days 14.61 , 26.55 and 57.51 , respectively. Note that these fits only include the known foreground reddening $\left(E_{B-V}=0.021\right)$, therefore the intrinsic slope of the SEDs of the nova eruption could be bluer.

The SEDs from the multi-band photometry are shown in Figure 8. The photometry taken at similar epochs as the spectra discussed above are broadly consistent with the power-laws derived from the spectra themselves, keeping in mind that as the nova fades, the broadband photometry becomes increasingly influenced by strong emission lines (e.g. Balmer and $\mathrm{O}$ I, and later [O III]). The photometry undoubtedly confirms that the dramatic change in the slope of the

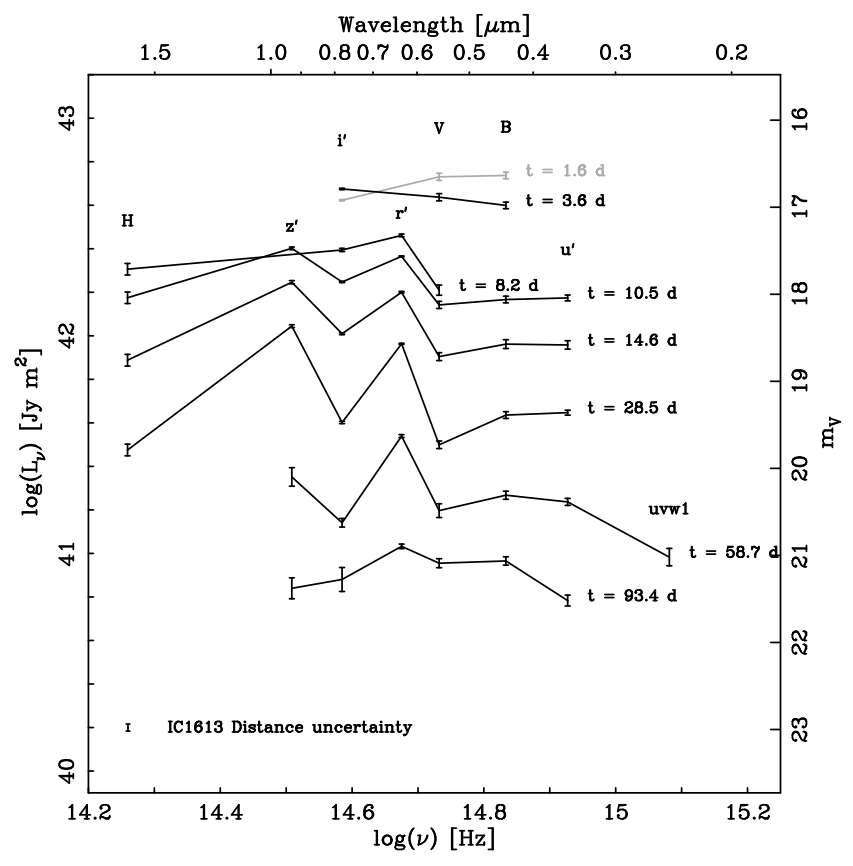

Figure 8. SEDs of Nova IC 16132015 from peak to 93.4 days post-discovery (around $m_{V}=4.4$ below peak). The extreme effect of the $\mathrm{H} \alpha$ emission on the $r^{\prime}$-band photometry can easily be seen. The size of the systematic uncertainty from the distance of IC 1613 is indicated near the bottom of the plot.

optical continuum emission between the spectra on day 1.59 and day 6.59 is real. Indeed a large change occurs between day 1.6 (i.e. the time of the first spectrum) and day 3.6.

\subsection{X-rays}

We do not detect X-ray emission from the nova between days 40-330 after eruption. The resulting luminosity upper limits, listed in Table 2 for each individual Swift observation, were typically below $5 \times 10^{37} \mathrm{erg} \mathrm{s}^{-1}$. This allows us to rule out a bright SSS under the conservative assumption of a $50 \mathrm{eV}$ black-body spectrum (the fastest novae are considerably hotter, see e.g. Osborne et al. 2011; Henze et al. 2014b; Page et al. 2015). Before day 40, the nova was still bright in UV and no X-rays would have been emitted (cf. Henze et al. 2015). An observing gap between days 160-260 (Table 2) is likely too short to hide a SSS phase: based on our experience with the M31 population, a nova with a SSS turn-on time of more than 160 days would be expected to be visible in X-rays for longer than 260 days (Henze et al. 2014a).

Combining all observations in Table $2(32.6 \mathrm{ks})$ we derive an upper limit of $4.5 \times 10^{-4} \mathrm{ct} \mathrm{s}^{-1}$, corresponding to a luminosity of $2.4 \times 10^{35} \mathrm{erg} \mathrm{s}^{-1}$. This is an order of magnitude lower than the observed luminosities of faint novae in M31 (Henze et al. 2010, 2011, 2014a). Since fainter novae are typically visible for longer (Henze et al. 2014a) we can rule out a low-luminosity SSS counterpart for the observed time range. Note that this upper limit would only be valid if such a low luminosity SSS was emitting over the whole time frame of the Swift observations. 


\subsection{The Progenitor Search}

The position of Nova IC 16132015 is not covered by Hubble Space Telescope data (which is ideal for such progenitor searches due to its high resolving power and large wavelength coverage), however at the distance of IC 1613, the most luminous quiescent systems will still be detectable in deep ground-based data. As we noted in Williams \& Darnley (2015), the nova appears very close to a $V=22.06, I=21.53$ magnitude source recorded at $01^{\mathrm{h}} 04^{\mathrm{m}} 43^{\mathrm{s}} .56+02^{\circ} 03^{\prime} 42^{\prime \prime} .0$ (J2000) in Udalski et al. (2001).

The field was observed with the Very Large Telescope (VLT) using the FOcal Reducer/low dispersion Spectrograph 2 (FORS2; Appenzeller et al. 1998) on 2012 Aug 20 under proposal 090.D-0009(A) and using the R_SPECIAL filter (effective wavelength $6550 \AA$ ). Using the method described in detail by Bode et al. (2009), Darnley et al. (2014) and Williams et al. (2014), we used reference stars in $r^{\prime}$-band LT eruption images to precisely determine the position of the nova in the archival data. This is shown in Figure 9. We also independently (using different reference stars) calculated the position of the nova in archival SDSS $g$-band OmegaCAM (Kuijken et al. 2002; Kuijken 2011) data taken at the $2.6 \mathrm{~m}$ VLT Survey Telescope (Arnaboldi et al. 1998) on 2014 Dec 17, using $V$-band LT data.

In the first archival image where the position was derived from the $r^{\prime}$-band LT eruption observations, the position of the nova is calculated to be $0^{\prime \prime} .09 \pm 0^{\prime \prime} .05$ south and $0^{\prime \prime} .21 \pm 0^{\prime \prime} .05$ east of the progenitor candidate. Using the errors on the positional transformation and the centroid on the nova/stellar source, implies an association between the two sources can be ruled out at the $4.1 \sigma$ level. In the second image we calculate the position of the nova to be $0^{\prime \prime} .05 \pm 0^{\prime \prime} .05$ north and $0^{\prime \prime} .25 \pm 0^{\prime \prime} .05$ east. From this it appears the progenitor candidate may have a small, but real offset (eastward) from the nova.

As a check for a systematic offset across the transformed field, we apply the positional transformation to 10 stars in close proximity to the nova (within $\sim 1^{\prime}$ ), that were not used in the calculation of the positional transformation itself. There is no evidence for a systematic offset in these sources, with the average $\mathrm{x} / \mathrm{y}$ offsets less than the standard deviation in the offsets. The standard deviation of these offsets however does indicate that it is possible the errors on the transformation are slightly underestimated. We therefore apply the average $\mathrm{x} / \mathrm{y}$ offsets (in the R_SPECIAL image) to the position of the nova. Using the standard deviation as the error indicates an association is still ruled out, but with a reduced $3 \sigma$ confidence. It is also worth noting that the transformed position of the source to the south-east of the nova (the brightest star seen in the left panel of Figure 9) is consistent within $1.1 \sigma$ (using the errors of the transformation itself) of that of the centroided position from the VLT image. If there were a systematic offset affecting the nova transformation, one would expect it to also be present in this very nearby $\left(\sim 5^{\prime \prime}\right.$ separation) source.

We therefore conclude that, despite the close proximity of the progenitor candidate, it is most likely simply a chance alignment. However, this should be confirmed by late-time spectroscopy. Novae retain strong Balmer emission for a significant time after eruption, but over time the optical spectrum becomes increasingly dominated by [O III] emission lines. If this progenitor candidate is indeed the luminous accretion disk of the pre-eruption nova, a quiescent spectrum may be expected to reveal narrow Balmer and He II emission. The lack of (broader) [O III] lines would confirm we are not observing an extended tail of the nova eruption.

\section{DISCUSSION}

A comparison of Nova IC 16132015 with other IC 1613 novae is not possible due to the lack of data for the 1954 and 1996 candidates. We can however compare it to other extragalactic novae residing in Local Group galaxies.

At $t_{2(V)}=13 \pm 2$ days, Nova IC 16132015 can be considered a fast-fading nova (Gaposchkin 1957). Comparing it to the cumulative $t_{2}$ distribution plot of M31 novae from Williams et al. (2016) would place it in the fastest $20 \%$ of novae. However a better comparison may be the LMC, and comparing the $t_{2}$ value to those in Table 2 from Shafter (2013) reveals that in this (albeit small) sample, novae significantly slower than Nova IC 16132015 are relatively rare. The low nova rate of the SMC, perhaps the best comparison to IC 1613, makes a comparison to the overall SMC population difficult. There are several SMC novae with good light curves (see e.g. Henize et al. 1954; de Laverny et al. 1998; Mróz et al. 2016) which display a broad range of decline times, and Nova IC 20162015 would certainly not seem out of place amongst these. Indeed, there have been some novae that evolved much more slowly than Nova IC 16132015 (e.g. Nova SMC 1994, de Laverny et al. 1998; Nova SMC 2001, Liller et al. 2004; Mróz et al. 2016).

The early decline spectra of Nova IC 16132015 are not typical of novae. In M31, around $80 \%$ of all novae belong to the Fe II class (Shafter et al. 2011). From the smaller sample size of LMC and M33 novae, a lower proportion (perhaps around $50 \%$ ) appear to be Fe II novae in these galaxies (Shafter et al. 2012; Shafter 2013). The hybrid spectroscopic class of novae can either evolve from one type to another or show both types simultaneously. Nova IC 16132015 shows both Fe II lines and N II in the early decline spectra, classifying it as a hybrid nova. It is worth noting that it is not unreasonable to expect hybrid novae that transition from one type to another to go through a phase which simultaneously shows both types, even if it is only short lived. The early decline spectral evolution shows many similarities to the hybrid Nova LMC 1988 No. 2 (see Sekiguchi et al. 1989 and Williams et al. 1991), although Nova IC 16132015 fades significantly more slowly, with Nova LMC 1988 No. 2 having a $t_{2}$ of around 5 days (Sekiguchi et al. 1989). The evolution of Nova IC 16132015 also appears similar to the Galactic nova V5114 Sagittarii, which had a similar $t_{2}(\sim 11$ days $)$ and showed somewhat similar spectroscopic evolution and associated velocities ( $\mathrm{H} \alpha \mathrm{FWHM} \sim 2000 \mathrm{~km} \mathrm{~s}^{-1}$; Ederoclite et al. 2006), although in this case the spectrum shortly after peak appears closer to a typical Fe II nova (Ederoclite et al. 2006) than Nova IC 16132015.

As the best Local Group galaxy with observed novae to compare IC 1613 to is the SMC, it is worth reviewing the spectroscopic information on SMC novae. Nova SMC 1951 was observed spectroscopically several times and clearly shows the Bowen blend emission complex (Henize et al. 1954). However most novae show this at later times, so an 

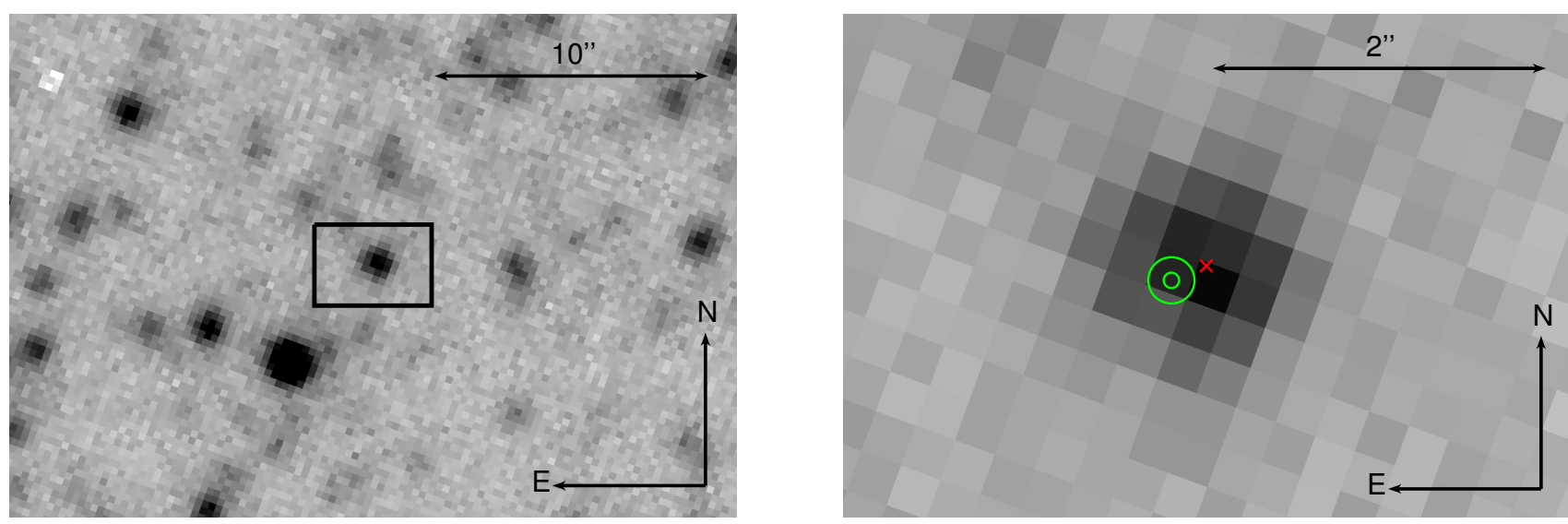

Figure 9. The position of the nova in pre-eruption data compared to the nearby resolved source. Left: The Nova IC 16132015 nova field imaged with FORS2 on the VLT using an R_SPECIAL filter on 2012 Aug 20. The black box indicates the zoomed in region shown in the right panel. Right: The same data as the left panel, with the $1 \sigma$ and $3 \sigma$ errors on the position of the nova (calculated from $r^{\prime}$-band LT eruption data) indicated by green circles and the position of the nearby resolved source indicated by a red ' $X$ '.

unambiguous spectroscopic type cannot be assigned. Nova SMC 1952 was observed two days after peak, and likely showed He I and He II emission (Smith 1954), classifying it as an $\mathrm{He} / \mathrm{N}$ nova. Nova SMC 2001 was an Fe II nova (Mason et al. 2005b). Nova SMC 2005 shows broad Fe II lines (Mason et al. 2005a), so is classed as an Fe IIb nova. Most recently, the first spectrum taken of Nova SMC 2016 was consistent with it being a member of the $\mathrm{He} / \mathrm{N}$ spectroscopic class (Williams \& Darnley 2016). We note that Nova SMC 2016 has extensive pan-chromatic coverage (see e.g. Kuin et al. 2016; Darnley \& Williams 2016; Page et al. 2016; Orio et al. 2016), which will be seen in forthcoming publication(s). The lack of early decline spectra of SMC novae prevents any conclusions being made on the proportion of novae that are Fe II novae or how Nova IC 16132015 compares to the SMC population.

There are clearly significant changes in the wavelength dependence of the underlying continuum in the early stages of the eruption, which is apparent from the spectra, where the slope of the continuum changes from $f_{\lambda} \propto \lambda^{-2.42 \pm 0.08}$ 1.59 days after discovery to $f_{\lambda} \propto \lambda^{-1.48 \pm 0.08} 6.59$ days after eruption. This is supported by the photometry where the nova shows a much redder colour 3.6 days after discovery compared to 1.6 days after discovery. In a nova eruption the $B, V$ and $i^{\prime \prime}$ magnitudes are not greatly affected by line emission until the latter stages.

If we ignore the first two photometry points (where we have already established the continuum has changed dramatically over a short time), the $B, V$, and $i^{\prime}$ light curves are relatively well described by a power law. We find $f_{B(\lambda)} \propto t^{-1.22}, f_{V(\lambda)} \propto t^{-1.28}$ and $f_{i^{\prime}(\lambda)} \propto t^{-1.46}$ (where $t$ is days since discovery), which indicates the nova is becoming increasingly blue as the eruption evolves (as seen between spectra 2 to 5$)$. However examining the $(B-V)$ colour evolution shows the picture is slightly more complex. The $(B-V)$ colour becomes lower until $m_{V} \sim 20$ (around day 30-40) and then turns around and $(B-V)$ begins increasing (getting redder). This behaviour is seen in other novae (see for example Figure 39 in Hachisu \& Kato 2014 and Figure 7 in Darnley et al. 2016). While this seems in general agreement with the power-law of the final spectrum becoming shallower, we also must note that while $B$ and $V$ magnitudes are not as influenced by line emission early in the eruption, during the nebular phase, lines such as [O III] become increasingly dominant (and thus affect the broadband colours, but are removed from the power-law fitting in Section 3.3).

The X-ray upper limits in Table 2 indicate that either the SSS phase had not started yet before day 330 or that the nova did not become visible in soft X-rays at all. If this were a M31 nova, then the fast $t_{2}$ and reasonably high expansion velocity would (consistently) predict a SSS turn-on time of about 50-90 days for a subset with similar properties (cf. Henze et al. 2014a). This is in agreement with the optical spectra indicating that by day 57 the nova had entered its nebular phase, where the ejecta would have become optically thin to X-rays. However, note that only a fraction of novae in the M31 reference sample showed SSS emission, which cannot be explained by observational coverage alone (Henze et al. 2011, 2014a).

If we assume that by day 57 the nuclear burning in the residual hydrogen envelope (i.e. the part that was not ejected) had already extinguished, then we can estimate an upper limit on the mass of this envelope. Following the approach described in Henze et al. (2014a, see also the relevant references therein) a SSS turn-off time of 57 days would correspond to a burned mass of $2.7 \times 10^{-7} \mathrm{M} \odot$. Such relatively low masses are rare, but have been estimated for a few fast novae from the M31 sample (Henze et al. 2014a). However, we stress the fact that for an individual object a large variety of factors, such as eruption geometry or inclination angle, can play a role in obscuring an existing SSS emission component.

Furthermore, there will of course be systematic differences between the nova samples of IC 1613 and M31, the latter of which we have learnt a great deal about nova population properties from. Different metallicities have been found to affect the average nova properties: see for instance the comparisons of M31 and LMC novae by della Valle \& Duerbeck (1993); Shafter (2013) and the theoretical models of (Hachisu \& Kato 2006; Kato et al. 2013). It remains unclear 
whether these systematics are large enough to affect the SSS phase of the nova significantly (e.g. to confine it to the narrow gap between days 160-260). Without further evidence we could only speculate on the specific causes for the SSS non detection, and we refrain from doing so.

\section{SUMMARY AND CONCLUSIONS}

We have presented detailed photometric and spectroscopic observations of the Nova IC 16132015 eruption, from the early optically thick stage, through the early decline and nebular phases. This is the first detailed study of a nova residing in an irregular dwarf galaxy beyond the much closer MCs. Here we summarise our observations and conclusions:

(i) We have undertaken a detailed observing campaign of Nova IC 1613 2015, with ground-based photometry and spectroscopy led by the LT, with further observations from LCO. We also obtained UV photometry and X-ray observations with Swift.

(ii) The light curve shows a relatively smooth decline and the nova is classified as a fast nova, with $t_{2(V)}=13 \pm 2$ and $t_{3(V)}=26 \pm 2$ days. The absolute peak magnitude of the nova is $M_{V}=-7.93 \pm 0.08$, which is typical for a classical nova.

(iii) The X-ray observations taken between 40-330 days after discovery detected no SSS emission associated with the nova.

(iv) The spectra show that the nova is a member of the 'hybrid' spectroscopic class, with it initially showing relatively strong Fe II lines and comparable N II lines. By the time it had declined by two magnitudes, the N II/N III features are significantly stronger than Fe II.

(v) One of the more unusual features is a strong emission line peaking at $\sim 6162 \AA$. We interpret this as likely due to O I $(6157 \AA)$, or possibly N II.

(vi) The FWHM of the $\mathrm{H} \alpha$ emission line is measured at around $1750 \mathrm{~km} \mathrm{~s}^{-1}$ and shows relatively little change over the course of the eruption.

(vii) The $\mathrm{H} \alpha / \mathrm{H} \beta$ ratio initially increases through the early decline spectra (due to self-absorption; peaking at $7.5 \pm 1.0$ ), before declining in the nebular spectrum. This implies the $z^{\prime}$-band light curve may be significantly influenced by a strong O I $8446 \AA$ emission line, which in turn is caused by $\mathrm{Ly} \beta$ fluorescence.

(viii) We also obtained a nebular spectrum of Nova IC 1613 2015, with [N II], [O I], [O II] and [O III] all detected. This makes it one of the first novae beyond the MCs to be observed in the nebular phase.

(ix) The first spectrum taken around peak shows a steep blue continuum of $f_{\lambda} \propto \lambda^{-2.42 \pm 0.08}$, similar to that expected from optically thick free-free emission, but also consistent with photospheric (black-body) emission. The second spectrum, shows a dramatic change in the continuum to $f_{\lambda} \propto \lambda^{-1.48 \pm 0.08}$. A sudden change in the underlying continuum between the two epochs is supported by the photometry.

(x) Despite the very close proximity of the nova to a stellar source, we find that this is most likely a chance alignment.

To further our understanding of how nova eruptions depend on the underlying stellar population it is important we take the opportunity to study novae occurring in significantly different environments than can be found in the usual targets of M31 and our own Galaxy.

\section{ACKNOWLEDGEMENTS}

We would like to thank the referee, Mike Shara, whose suggestions helped improve this paper. SCW acknowledges a visiting research fellowship at Liverpool John Moores University (LJMU). MH acknowledges the support of the Spanish Ministry of Economy and Competitiveness (MINECO) under the grant FDPI-2013-16933 as well as the support of the Generalitat de Catalunya/CERCA programme. The Liverpool Telescope is operated on the island of La Palma by LJMU in the Spanish Observatorio del Roque de los Muchachos of the Instituto de Astrofisica de Canarias with financial support from the UK Science and Technology Facilities Council. This work makes use of observations from the LCO network. This work made use of data supplied by the UK Swift Science Data Centre at the University of Leicester. Based in part on data obtained from the ESO Science Archive Facility under request numbers scw233242 and scw233245.

\section{REFERENCES}

Ahn C. P., et al., 2012, ApJS, 203, 21

Anupama G. C., Duerbeck H. W., Prabhu T. P., Jain S. K., 1992, A\&A, 263, 87

Appenzeller I., et al., 1998, The Messenger, 94, 1

Arnaboldi M., Capaccioli M., Mancini D., Rafanelli P., Scaramella R., Sedmak G., Vettolani G. P., 1998, The Messenger, 93, 30 Arnould M., Norgaard H., 1975, A\&A, 42, 55

Barnsley R. M., Jermak H. E., Steele I. A., Smith R. J., Bates S. D., Mottram C. J., 2016, Journal of Astronomical Telescopes, Instruments, and Systems, 2, 015002

Baschek B., 1964, PASP, 76, 22

Beck H. K. B., Hauschildt P. H., Gail H.-P., Sedlmayr E., 1995, A\&A, 294, 195

Bode M. F., Evans A., 2008, Classical Novae

Bode M. F., Darnley M. J., Shafter A. W., Page K. L., Smirnova O., Anupama G. C., Hilton T., 2009, ApJ, 705, 1056

Bouret J.-C., Lanz T., Hillier D. J., Martins F., Marcolino W. L. F., Depagne E., 2015, MNRAS, 449, 1545

Bowen I. S., 1947, PASP, 59, 196

Brown T. M., et al., 2013, PASP, 125, 1031

Burrows D. N., et al., 2005, Space Sci. Rev., 120, 165

Cardelli J. A., Clayton G. C., Mathis J. S., 1989, ApJ, 345, 245

Cutri R. M., et al., 2003, VizieR Online Data Catalog, 2246, 0

Darnley M. J., Williams S. C., 2016, The Astronomer's Telegram, 9688

Darnley M. J., et al., 2006, MNRAS, 369, 257

Darnley M. J., Ribeiro V. A. R. M., Bode M. F., Hounsell R. A., Williams R. P., 2012, ApJ, 746, 61

Darnley M. J., Williams S. C., Bode M. F., Henze M., Ness J.-U., Shafter A. W., Hornoch K., Votruba V., 2014, A\&A, 563, L9 Darnley M. J., et al., 2016, ApJ, 833, 149

Dickey J. M., Lockman F. J., 1990, ARA\&A, 28, 215

Ederoclite A., et al., 2006, A\&A, 459, 875

Evans A., Tyne V. H., Smith O., Geballe T. R., Rawlings J. M. C., Eyres S. P. S., 2005, MNRAS, 360, 1483

Evans P. A., et al., 2009, MNRAS, 397, 1177

Ferland G. J., 1978, ApJ, 219, 589

Ferland G., Netzer H., 1979, ApJ, 229, 274 
Ferland G. J., Shields G. A., Netzer H., 1979, ApJ, 232, 382

Ferland G. J., Lambert D. L., Woodman J. H., 1986, ApJS, 60, 375

Ferrarese L., Côté P., Jordán A., 2003, ApJ, 599, 1302

Filippenko A. V., Li W. D., Treffers R. R., Modjaz M., 2001, in Paczynski B., Chen W.-P., Lemme C., eds, Astronomical Society of the Pacific Conference Series Vol. 246, IAU Colloq. 183: Small Telescope Astronomy on Global Scales. p. 121

Gaposchkin C. H. P., 1957, The galactic novae.

Garcia M., Herrero A., Vicente B., Castro N., Corral L. J., Rosenberg A., Monelli M., 2009, A\&A, 502, 1015

Garcia M., Herrero A., Najarro F., Lennon D. J., Alejandro Urbaneja M., 2014, ApJ, 788, 64

Gehrels N., et al., 2004, ApJ, 611, 1005

Hachisu I., Kato M., 2006, ApJS, 167, 59

Hachisu I., Kato M., 2014, ApJ, 785, 97

Halevi G., Zheng W., Filippenko A. V., 2015, Central Bureau Electronic Telegrams, 4186, 1

Henize K. G., Hoffleit D., McKibben Nail V., 1954, Proceedings of the National Academy of Science, 40, 365

Henze M., et al., 2010, A\&A, 523, A89

Henze M., et al., 2011, A\&A, 533, A52

Henze M., et al., 2014a, A\&A, 563, A2

Henze M., Ness J.-U., Darnley M. J., Bode M. F., Williams S. C., Shafter A. W., Kato M., Hachisu I., 2014b, A\&A, 563, L8

Henze M., et al., 2015, A\&A, 580, A46

Hernanz M., Jose J., Coc A., Isern J., 1996, ApJ, 465, L27

Hillebrandt W., Niemeyer J. C., 2000, ARA\&A, 38, 191

Hillman Y., Prialnik D., Kovetz A., Shara M. M., 2016, ApJ, 819, 168

Hoyle F., Fowler W. A., 1960, ApJ, 132, 565

Huggins W., 1866, MNRAS, 26, 275

Iijima T., Esenoglu H. H., 2003, A\&A, 404, 997

Izzo L., et al., 2015, ApJ, 808, L14

Jordi K., Grebel E. K., Ammon K., 2006, A\&A, 460, 339

Kastner S. O., Bhatia A. K., 1995, ApJ, 439, 346

Kato M., Hachisu I., Henze M., 2013, ApJ, 779, 19

King J. Y., Modjaz M., Li W. D., 1999, IAU Circ., 7287, 2

Kirby E. N., Bullock J. S., Boylan-Kolchin M., Kaplinghat M., Cohen J. G., 2014, MNRAS, 439, 1015

Kogure T., 1961, PASJ, 13, 335

Kraft R. P., 1964, ApJ, 139, 457

Kraft R. P., Burrows D. N., Nousek J. A., 1991, ApJ, 374, 344

Krautter J., Oegelman H., Starrfield S., Wichmann R., Pfeffermann E., 1996, ApJ, 456, 788

Kuijken K., 2011, The Messenger, 146, 8

Kuijken K., et al., 2002, The Messenger, 110, 15

Kuin N. P. M., Page K. L., Williams S. C., Darnley M. J., Shore S. N., Walter F. M., 2016, The Astronomer's Telegram, 9635

Kurtev R., Georgiev L., Borissova J., Li W. D., Filippenko A. V., Treffers R. R., 2001, A\&A, 378, 449

Liller W., Shida R. Y., Jones A. F., 2004, Information Bulletin on Variable Stars, 5582

Mantegazza L., Antonello E., Fugazza D., Bossi M., Covino S., 2001, A\&A, 367, 759

Maoz D., Mannucci F., Nelemans G., 2014, ARA\&A, 52, 107

Mason E., Brandeker A., Ederoclite A., Della Valle M., 2005a, Central Bureau Electronic Telegrams, 195

Mason E., Della Valle M., Gilmozzi R., Lo Curto G., Williams R. E., 2005b, A\&A, 435, 1031

Meinel A. B., 1963, ApJ, 137, 834

Menzies J. W., Whitelock P. A., Feast M. W., 2015, MNRAS, 452,910

Molaro P., Izzo L., Mason E., Bonifacio P., Della Valle M., 2016, MNRAS, 463, L117

Moore C. E., 1945, Contributions from the Princeton University Observatory, 20, 1

Mróz P., et al., 2016, ApJS, 222, 9
Munari U., et al., 2014, MNRAS, 440, 3402

Neill J. D., Shara M. M., 2004, AJ, 127, 816

Netzer H., 1975, MNRAS, 171, 395

Nomoto K., Thielemann F.-K., Yokoi K., 1984, ApJ, 286, 644

Nugent P. E., et al., 2011, Nature, 480, 344

Oke J. B., 1990, AJ, 99, 1621

Orio M., Behar E., Rauch T., Zemk P., 2016, The Astronomer's Telegram, 9810

Osborne J. P., et al., 2011, ApJ, 727, 124

Page K. L., et al., 2015, MNRAS, 454, 3108

Page K., Osborne J., Kuin P., Shore S., Williams S., Darnley M. J., 2016, The Astronomer's Telegram, 9733

Piascik A. S., Steele I. A., Bates S. D., Mottram C. J., Smith R. J., Barnsley R. M., Bolton B., 2014, in Society of PhotoOptical Instrumentation Engineers (SPIE) Conference Series. p. 8, doi:10.1117/12.2055117

Pietrzyński G., Gieren W., Soszyński I., Bresolin F., Kudritzki R.-P., Dall'Ora M., Storm J., Bono G., 2006, ApJ, 642, 216

Poole T. S., et al., 2008, MNRAS, 383, 627

Prialnik D., 1986, ApJ, 310, 222

Roming P. W. A., et al., 2005, Space Sci. Rev., 120, 95

Sandage A., 1971, ApJ, 166, 13

Schlafly E. F., Finkbeiner D. P., 2011, ApJ, 737, 103

Scowcroft V., Freedman W. L., Madore B. F., Monson A. J., Persson S. E., Seibert M., Rigby J. R., Melbourne J., 2013, ApJ, 773, 106

Sekiguchi K., Kilkenny D., Winkler H., Doyle J. G., 1989, MNRAS, 241, 827

Shafter A. W., 2013, AJ, 145, 117

Shafter A. W., Rau A., Quimby R. M., Kasliwal M. M., Bode M. F., Darnley M. J., Misselt K. A., 2009, ApJ, 690, 1148

Shafter A. W., et al., 2011, ApJ, 734, 12

Shafter A. W., Darnley M. J., Bode M. F., Ciardullo R., 2012, ApJ, 752, 156

Shara M. M., et al., 2016, ApJS, 227, 1

Shara M. M., et al., 2017, preprint, (arXiv:1702.06988)

Shields G. A., 1974, ApJ, 191, 309

Shore S. N., et al., 2013, The Astronomer's Telegram, 5378

Skillman E. D., et al., 2014, ApJ, 786, 44

Smith H. J., 1954, AJ, 59, 193

Starrfield S., Truran J. W., Sparks W. M., Kutter G. S., 1972, ApJ, 176, 169

Starrfield S., Truran J. W., Sparks W. M., Arnould M., 1978, ApJ, 222, 600

Steele I. A., et al., 2004, in Oschmann Jr. J. M., ed., Society of Photo-Optical Instrumentation Engineers (SPIE) Conference Series Vol. 5489, Society of Photo-Optical Instrumentation Engineers (SPIE) Conference Series. pp 679-692, doi: $10.1117 / 12.551456$

Surina F., Hounsell R. A., Bode M. F., Darnley M. J., Harman D. J., Walter F. M., 2014, AJ, 147, 107

Tajitsu A., Sadakane K., Naito H., Arai A., Aoki W., 2015, Nature, 518,381

Tajitsu A., Sadakane K., Naito H., Arai A., Kawakita H., Aoki W., 2016, ApJ, 818, 191

Tody D., 1986, in Crawford D. L., ed., Society of Photo-Optical Instrumentation Engineers (SPIE) Conference Series Vol. 627, Instrumentation in astronomy VI. p. 733

Udalski A., Wyrzykowski L., Pietrzynski G., Szewczyk O., Szymanski M., Kubiak M., Soszynski I., Zebrun K., 2001, Acta Astron., 51, 221

Walker M. F., 1954, PASP, 66, 230

Whelan J., Iben Jr. I., 1973, ApJ, 186, 1007

Williams R. E., 1992, AJ, 104, 725

Williams R., 2012, AJ, 144, 98

Williams S. C., Darnley M. J., 2015, The Astronomer's Telegram, 8061 
Williams S. C., Darnley M. J., 2016, The Astronomer's Telegram, 9628

Williams R. E., Hamuy M., Phillips M. M., Heathcote S. R., Wells L., Navarrete M., 1991, ApJ, 376, 721

Williams S. C., Darnley M. J., Bode M. F., Keen A., Shafter A. W., 2014, ApJS, 213, 10

Williams S. C., Darnley M. J., Bode M. F., Shafter A. W., 2016, ApJ, 817, 143

Woudt P. A., Ribeiro V. A. R. M., eds, 2014, Stella Novae: Past and Future Decades Astronomical Society of the Pacific Conference Series Vol. 490

Wright W. H., 1940, Publications of Lick Observatory, 14, 27

Wright A. E., Barlow M. J., 1975, MNRAS, 170, 41

de Laverny P., et al., 1998, A\&A, 335, L93

della Valle M., Duerbeck H. W., 1993, A\&A, 271, 175

van den Bergh S., 2000, The Galaxies of the Local Group. Cambridge

\section{APPENDIX A: NUV, OPTICAL, AND NIR \\ PHOTOMETRY OF NOVA IC 16132015}

This paper has been typeset from a $\mathrm{TE}_{\mathrm{E}} / \mathrm{LAT}_{\mathrm{EX}}$ file prepared by the author. 
Table A1. Near-UV, optical, and near-IR photometry of Nova IC 1613 2015. These data have not been corrected for any reddening.

\begin{tabular}{|c|c|c|c|c|c|}
\hline Date $[\mathrm{UT}]$ & $t$ [days] & Telescope \& Instrument & Exposure $[\mathrm{s}]$ & Filter & Photometry \\
\hline 2016 Sep 20.993 & 10.513 & LT IO:O & 180 & $u^{\prime}$ & $18.078 \pm 0.033$ \\
\hline 2016 Sep 21.978 & 11.498 & LT IO:O & 120 & $u^{\prime}$ & $17.954 \pm 0.038$ \\
\hline 2016 Sep 25.046 & 14.566 & LT IO:O & 180 & $u^{\prime}$ & $18.618 \pm 0.048$ \\
\hline 2016 Sep 25.948 & 15.468 & LT IO:O & 180 & $u^{\prime}$ & $18.653 \pm 0.064$ \\
\hline 2016 Sep 27.036 & 16.556 & LT IO:O & 360 & $u^{\prime}$ & $18.583 \pm 0.053$ \\
\hline 2016 Sep 30.962 & 20.482 & LT IO:O & 360 & $u^{\prime}$ & $18.894 \pm 0.044$ \\
\hline 2016 Oct 06.009 & 25.529 & LT IO:O & 240 & $u^{\prime}$ & $19.181 \pm 0.030$ \\
\hline 2016 Oct 08.078 & 27.598 & LT IO:O & 360 & $u^{\prime}$ & $19.353 \pm 0.029$ \\
\hline 2016 Oct 09.014 & 28.534 & LT IO:O & 360 & $u^{\prime}$ & $19.396 \pm 0.028$ \\
\hline 2016 Oct 13.014 & 32.534 & LT IO:O & 360 & $u^{\prime}$ & $19.527 \pm 0.031$ \\
\hline 2016 Nov 03.995 & 54.515 & LT IO:O & 360 & $u^{\prime}$ & $20.317 \pm 0.036$ \\
\hline 2016 Nov 07.983 & 58.503 & LT IO:O & 360 & $u^{\prime}$ & $20.421 \pm 0.040$ \\
\hline 2016 Nov 15.967 & 66.487 & LT IO:O & 360 & $u^{\prime}$ & $20.741 \pm 0.040$ \\
\hline 2016 Dec 12.868 & 93.388 & LT IO:O & 360 & $u^{\prime}$ & $21.554 \pm 0.062$ \\
\hline 2016 Sep 12.087 & 1.607 & LT IO:O & 180 & $B$ & $16.827 \pm 0.038$ \\
\hline 2016 Sep 14.043 & 3.563 & LT IO:O & 60 & $B$ & $17.171 \pm 0.038$ \\
\hline 2016 Sep 17.114 & 6.634 & LT IO:O & 60 & $B$ & $17.675 \pm 0.038$ \\
\hline 2016 Sep 20.996 & 10.516 & LT IO:O & 180 & $B$ & $18.252 \pm 0.038$ \\
\hline 2016 Sep 21.981 & 11.501 & LT IO:O & 120 & $B$ & $18.226 \pm 0.039$ \\
\hline 2016 Sep 24.606 & 14.126 & LCO $2 \mathrm{~m}$ Spectral & 360 & $B$ & $18.927 \pm 0.068$ \\
\hline 2016 Sep 25.049 & 14.569 & LT IO:O & 180 & $B$ & $18.764 \pm 0.051$ \\
\hline 2016 Sep 25.951 & 15.471 & LT IO:O & 180 & $B$ & $18.925 \pm 0.047$ \\
\hline 2016 Sep 27.057 & 16.577 & LT IO:O & 180 & $B$ & $18.816 \pm 0.050$ \\
\hline 2016 Sep 29.929 & 19.449 & LT IO:O & 180 & $B$ & $18.932 \pm 0.055$ \\
\hline 2016 Oct 3.576 & 23.096 & LCO 2 m Spectral & 360 & $B$ & $19.461 \pm 0.045$ \\
\hline 2016 Oct 05.994 & 25.514 & LT IO:O & 180 & $B$ & $19.430 \pm 0.039$ \\
\hline 2016 Oct 08.062 & 27.582 & LT IO:O & 180 & $B$ & $19.567 \pm 0.039$ \\
\hline 2016 Oct 08.999 & 28.519 & LT IO:O & 180 & $B$ & $19.579 \pm 0.039$ \\
\hline 2016 Oct 10.920 & 30.440 & LT IO:O & 180 & $B$ & $19.671 \pm 0.042$ \\
\hline 2016 Oct 12.999 & 32.519 & LT IO:O & 180 & $B$ & $19.803 \pm 0.040$ \\
\hline 2016 Oct 18.969 & 38.489 & LT IO:O & 180 & $B$ & $20.041 \pm 0.065$ \\
\hline 2016 Oct 20.530 & 40.050 & LCO $2 \mathrm{~m}$ Spectral & 360 & $B$ & $20.154 \pm 0.073$ \\
\hline 2016 Oct 30.038 & 49.558 & LT IO:O & 180 & $B$ & $20.275 \pm 0.063$ \\
\hline 2016 Nov 03.980 & 54.500 & LT IO:O & 180 & $B$ & $20.362 \pm 0.043$ \\
\hline 2016 Nov 07.969 & 58.489 & LT IO:O & 180 & $B$ & $20.500 \pm 0.046$ \\
\hline 2016 Nov 15.951 & 66.471 & LT IO:O & 120 & $B$ & $20.698 \pm 0.052$ \\
\hline 2016 Dec 12.845 & 93.365 & LT IO:O & 240 & $B$ & $21.255 \pm 0.048$ \\
\hline 2016 Sep 12.090 & 1.610 & LT IO:O & 180 & $V$ & $16.651 \pm 0.041$ \\
\hline 2016 Sep 14.044 & 3.564 & LT IO:O & 60 & $V$ & $16.885 \pm 0.041$ \\
\hline 2016 Sep 17.115 & 6.635 & LT IO:O & 60 & $V$ & $17.493 \pm 0.041$ \\
\hline 2016 Sep 18.499 & 8.020 & LCO 2 m Spectral & 360 & $V$ & $17.953 \pm 0.058$ \\
\hline 2016 Sep 20.978 & 10.498 & LT IO:O & 180 & $V$ & $18.122 \pm 0.041$ \\
\hline 2016 Sep 21.984 & 11.504 & LT IO:O & 180 & $V$ & $18.118 \pm 0.037$ \\
\hline 2016 Sep 24.611 & 14.131 & LCO $2 \mathrm{~m}$ Spectral & 360 & $V$ & $18.722 \pm 0.056$ \\
\hline 2016 Sep 25.051 & 14.571 & LT IO:O & 180 & $V$ & $18.716 \pm 0.045$ \\
\hline 2016 Sep 25.954 & 15.474 & LT IO:O & 120 & V & $18.767 \pm 0.051$ \\
\hline 2016 Sep 27.061 & 16.581 & LT IO:O & 180 & $V$ & $18.749 \pm 0.047$ \\
\hline 2016 Sep 29.934 & 19.454 & LT IO:O & 180 & $V$ & $19.012 \pm 0.051$ \\
\hline 2016 Sep 30.949 & 20.469 & LT IO:O & 180 & $V$ & $19.078 \pm 0.045$ \\
\hline 2016 Oct 03.580 & 23.100 & LCO 2 m Spectral & 360 & $V$ & $19.418 \pm 0.048$ \\
\hline 2016 Oct 05.996 & 25.516 & LT IO:O & 180 & $V$ & $19.457 \pm 0.042$ \\
\hline 2016 Oct 09.001 & 28.521 & LT IO:O & 180 & $V$ & $19.729 \pm 0.043$ \\
\hline 2016 Oct 10.923 & 30.443 & LT IO:O & 180 & $V$ & $19.791 \pm 0.044$ \\
\hline 2016 Oct 13.002 & 32.522 & LT IO:O & 180 & $V$ & $19.939 \pm 0.044$ \\
\hline 2016 Oct 18.974 & 38.494 & LT IO:O & 120 & $V$ & $20.077 \pm 0.050$ \\
\hline 2016 Oct 20.536 & 40.056 & LCO 2 m Spectral & 360 & $V$ & $20.147 \pm 0.077$ \\
\hline 2016 Oct 30.041 & 49.561 & LT IO:O & 180 & $V$ & $20.343 \pm 0.101$ \\
\hline 2016 Nov 03.983 & 54.503 & LT IO:O & 180 & V & $20.588 \pm 0.060$ \\
\hline 2016 Nov 07.972 & 58.492 & LT IO:O & 180 & $V$ & $20.485 \pm 0.079$ \\
\hline 2016 Nov 15.954 & 66.474 & LT IO:O & 180 & $V$ & $20.696 \pm 0.069$ \\
\hline 2016 Dec 12.849 & 93.369 & LT IO:O & 300 & $V$ & $21.089 \pm 0.052$ \\
\hline
\end{tabular}


Table A1 - continued Near-UV, optical, and near-IR photometry of Nova IC 1613 2015. These data have not been corrected for reddening.

\begin{tabular}{|c|c|c|c|c|c|}
\hline Date [UT] & $t$ [days] & Telescope \& Instrument & Exposure [s] & Filter & Photometry \\
\hline 2016 Sep 18.503 & 8.023 & LCO $2 \mathrm{~m}$ Spectral & 180 & $r^{\prime}$ & $17.309 \pm 0.015$ \\
\hline 2016 Sep 21.002 & 10.522 & LT IO:O & 180 & $r^{\prime}$ & $17.550 \pm 0.005$ \\
\hline 2016 Sep 21.987 & 11.507 & LT IO:O & 120 & $r^{\prime}$ & $17.537 \pm 0.006$ \\
\hline 2016 Sep 24.616 & 14.136 & LCO $2 \mathrm{~m}$ Spectral & 360 & $r^{\prime}$ & $17.967 \pm 0.013$ \\
\hline 2016 Sep 25.054 & 14.574 & LT IO:O & 180 & $r^{\prime}$ & $17.962 \pm 0.009$ \\
\hline 2016 Sep 25.957 & 15.477 & LT IO:O & 180 & $r^{\prime}$ & $18.032 \pm 0.011$ \\
\hline 2016 Sep 27.062 & 16.582 & LT IO:O & 180 & $r^{\prime}$ & $18.051 \pm 0.012$ \\
\hline 2016 Sep 29.938 & 19.458 & LT IO:O & 180 & $r^{\prime}$ & $18.277 \pm 0.030$ \\
\hline 2016 Sep 30.952 & 20.472 & LT IO:O & 180 & $r^{\prime}$ & $18.314 \pm 0.010$ \\
\hline 2016 Oct 03.585 & 23.105 & LCO $2 \mathrm{~m}$ Spectral & 360 & $r^{\prime}$ & $18.371 \pm 0.009$ \\
\hline 2016 Oct 05.999 & 25.519 & LT IO:O & 60 & $r^{\prime}$ & $18.453 \pm 0.009$ \\
\hline 2016 Oct 08.068 & 27.588 & LT IO:O & 180 & $r^{\prime}$ & $18.532 \pm 0.007$ \\
\hline 2016 Oct 09.004 & 28.524 & LT IO:O & 180 & $r^{\prime}$ & $18.554 \pm 0.006$ \\
\hline 2016 Oct 10.925 & 30.445 & LT IO:O & 120 & $r^{\prime}$ & $18.579 \pm 0.009$ \\
\hline 2016 Oct 13.004 & 32.524 & LT IO:O & 180 & $r^{\prime}$ & $18.651 \pm 0.007$ \\
\hline 2016 Oct 18.976 & 38.496 & LT IO:O & 180 & $r^{\prime}$ & $18.845 \pm 0.012$ \\
\hline 2016 Oct 20.541 & 40.061 & LCO $2 \mathrm{~m}$ Spectral & 360 & $r^{\prime}$ & $18.892 \pm 0.015$ \\
\hline 2016 Oct 30.044 & 49.564 & LT IO:O & 180 & $r^{\prime}$ & $19.269 \pm 0.017$ \\
\hline 2016 Nov 03.985 & 54.505 & LT IO:O & 60 & $r^{\prime}$ & $19.487 \pm 0.021$ \\
\hline 2016 Nov 07.974 & 58.494 & LT IO:O & 180 & $r^{\prime}$ & $19.614 \pm 0.016$ \\
\hline 2016 Nov 15.957 & 66.477 & LT IO:O & 180 & $r^{\prime}$ & $20.003 \pm 0.016$ \\
\hline 2016 Dec 12.853 & 93.373 & LT IO:O & 240 & $r^{\prime}$ & $20.881 \pm 0.026$ \\
\hline 2016 Sep 12.094 & 1.614 & LT IO:O & 360 & $i^{\prime}$ & $16.891 \pm 0.007$ \\
\hline 2016 Sep 14.045 & 3.565 & LT IO:O & 60 & $i^{\prime}$ & $16.762 \pm 0.008$ \\
\hline 2016 Sep 17.116 & 6.636 & LT IO:O & 60 & $i^{\prime}$ & $17.185 \pm 0.008$ \\
\hline 2016 Sep 18.506 & 8.026 & LCO $2 \mathrm{~m}$ Spectral & 180 & $i^{\prime}$ & $17.462 \pm 0.018$ \\
\hline 2016 Sep 21.005 & 10.525 & LT IO:O & 180 & $i^{\prime}$ & $17.830 \pm 0.007$ \\
\hline 2016 Sep 21.990 & 11.510 & LT IO:O & 180 & $i^{\prime}$ & $17.877 \pm 0.007$ \\
\hline 2016 Sep 24.621 & 14.141 & LCO $2 \mathrm{~m}$ Spectral & 360 & $i^{\prime}$ & $18.427 \pm 0.021$ \\
\hline 2016 Sep 25.057 & 14.577 & LT IO:O & 180 & $i^{\prime}$ & $18.425 \pm 0.011$ \\
\hline 2016 Sep 25.960 & 15.480 & LT IO:O & 180 & $i^{\prime}$ & $18.509 \pm 0.015$ \\
\hline 2016 Sep 27.065 & 16.585 & LT IO:O & 180 & $i^{\prime}$ & $18.553 \pm 0.019$ \\
\hline 2016 Sep 29.941 & 19.461 & LT IO:O & 180 & $i^{\prime}$ & $18.814 \pm 0.021$ \\
\hline 2016 Sep 30.955 & 20.475 & LT IO:O & 180 & $i^{\prime}$ & $18.899 \pm 0.017$ \\
\hline 2016 Oct 03.590 & 23.110 & LCO $2 \mathrm{~m}$ Spectral & 360 & $i^{\prime}$ & $19.144 \pm 0.019$ \\
\hline 2016 Oct 06.002 & 25.522 & LT IO:O & 180 & $i^{\prime}$ & $19.243 \pm 0.012$ \\
\hline 2016 Oct 08.071 & 27.591 & LT IO:O & 180 & $i^{\prime}$ & $19.400 \pm 0.013$ \\
\hline 2016 Oct 09.007 & 28.527 & LT IO:O & 180 & $i^{\prime}$ & $19.444 \pm 0.013$ \\
\hline 2016 Oct 10.928 & 30.448 & LT IO:O & 180 & $i^{\prime}$ & $19.529 \pm 0.019$ \\
\hline 2016 Oct 13.007 & 32.527 & LT IO:O & 180 & $i^{\prime}$ & $19.642 \pm 0.016$ \\
\hline 2016 Oct 20.544 & 40.064 & LCO $2 \mathrm{~m}$ Spectral & 360 & $i^{\prime}$ & $20.013 \pm 0.051$ \\
\hline 2016 Oct 30.047 & 49.567 & LT IO:O & 180 & $i^{\prime}$ & $20.415 \pm 0.047$ \\
\hline 2016 Nov 03.988 & 54.508 & LT IO:O & 180 & $i^{\prime}$ & $20.548 \pm 0.038$ \\
\hline 2016 Nov 07.977 & 58.497 & LT IO:O & 180 & $i^{\prime}$ & $20.596 \pm 0.049$ \\
\hline 2016 Nov 15.960 & 66.480 & LT IO:O & 180 & $i^{\prime}$ & $20.832 \pm 0.049$ \\
\hline 2016 Dec 12.866 & 93.386 & LT IO:O & 300 & $i^{\prime}$ & $21.248 \pm 0.138$ \\
\hline 2016 Sep 21.008 & 10.528 & LT IO:O & 180 & $z^{\prime}$ & $17.431 \pm 0.015$ \\
\hline 2016 Sep 21.993 & 11.513 & LT IO:O & 120 & $z$ & $17.443 \pm 0.015$ \\
\hline 2016 Sep 25.060 & 14.580 & LT IO:O & 180 & $z$ & $17.820 \pm 0.018$ \\
\hline 2016 Sep 25.963 & 15.483 & LT IO:O & 180 & $z$ & $17.879 \pm 0.027$ \\
\hline 2016 Sep 27.068 & 16.588 & LT IO:O & 180 & $z$ & $17.904 \pm 0.022$ \\
\hline 2016 Sep 27.940 & 17.460 & LT IO:O & 180 & $z$ & $17.990 \pm 0.039$ \\
\hline 2016 Sep 29.944 & 19.464 & LT IO:O & 180 & $z$ & $18.065 \pm 0.023$ \\
\hline 2016 Sep 30.958 & 20.478 & LT IO:O & 180 & $z$ & $18.078 \pm 0.019$ \\
\hline 2016 Oct 06.005 & 25.525 & LT IO:O & 120 & $z$ & $18.172 \pm 0.018$ \\
\hline 2016 Oct 08.074 & 27.594 & LT IO:O & 120 & $z$ & $18.322 \pm 0.019$ \\
\hline 2016 Oct 09.010 & 28.530 & LT IO:O & 180 & $z$ & $18.337 \pm 0.016$ \\
\hline 2016 Oct 13.010 & 32.530 & LT IO:O & 180 & $z$ & $18.556 \pm 0.018$ \\
\hline 2016 Oct 18.981 & 38.501 & LT IO:O & 180 & $z$ & $18.950 \pm 0.050$ \\
\hline 2016 Oct 30.050 & 49.570 & LT IO:O & 120 & $z$ & $19.689 \pm 0.089$ \\
\hline 2016 Nov 03.992 & 54.512 & LT IO:O & 180 & $z$ & $19.940 \pm 0.085$ \\
\hline
\end{tabular}


Table A1 - continued Near-UV, optical, and near-IR photometry of Nova IC 1613 2015. These data have not been corrected for reddening.

\begin{tabular}{llllll}
\hline Date [UT] & $t$ [days] & Telescope \& Instrument & Exposure [s] & Filter & Photometry \\
\hline 2016 Nov 07.980 & 58.500 & LT IO:O & 180 & $z$ & $20.057 \pm 0.106$ \\
2016 Nov 15.963 & 66.483 & LT IO:O & 180 & $z$ & $20.536 \pm 0.084$ \\
2016 Dec 12.863 & 93.383 & LT IO:O & 300 & $z$ & $21.366 \pm 0.120$ \\
\hline 2016 Sep 19.191 & 8.711 & LT IO:I & 538 & $H$ & $16.598 \pm 0.103$ \\
2016 Sep 21.043 & 10.563 & LT IO:I & 538 & $H$ & $16.991 \pm 0.117$ \\
2016 Sep 25.066 & 14.586 & LT IO:I & 538 & $H$ & $17.678 \pm 0.102$ \\
2016 Oct 08.965 & 28.485 & LT IO:I & 538 & $H$ & $18.772 \pm 0.143$ \\
\hline
\end{tabular}

